\title{
Exponential Mixing for Stochastic PDEs: The Non-Additive Case.
}

\author{
CYRIL ODASSO \\ ECOLE NORMALE SUPÉRIEURE DE CACHAN, ANTENNE DE BRETAGNE, \\ AVENUE ROBERT SCHUMAN, CAMPUS DE KER LANN, 35170 BRUZ (FRANCE). \\ AND \\ IRMAR, UMR 6625 DU CNRS, CAMPUS DE BEAULIEU, 35042 RENNES CEDEX
}

(FRANCE)

\begin{abstract}
We establish a general criterion which ensures exponential mixing of parabolic Stochastic Partial Differential Equations (SPDE) driven by a non additive noise which is white in time and smooth in space. We apply this criterion on two representative examples: 2D Navier-Stokes (NS) equations and Complex Ginzburg-Landau (CGL) equation with a locally Lipschitz noise. Due to the possible degeneracy of the noise, Doob theorem cannot be applied. Hence a coupling method is used in the spirit of 9, 23 and 26.

Previous results require assumptions on the covariance of the noise which might seem restrictive and artificial. For instance, for NS and CGL, the covariance operator is supposed to be diagonal in the eigenbasis of the Laplacian and not depending on the high modes of the solutions. The method developed in the present paper gets rid of such assumptions and only requires that the range of the covariance operator contains the low modes.
\end{abstract}

Key words: Two-dimensional Navier-Stokes equations, Complex Ginzburg-Landau equations, Markov transition semi-group, invariant measure, ergodicity, coupling method, Girsanov Formula, expectational Foias-Prodi estimate.

\section{INTRODUCTION}

We investigate ergodic properties of parabolic Stochastic Partial Differential Equations (SPDE) driven by a noise which is white in time and smooth in space. Such systems are difficult to handle with the standard theory because the phase spaces are infinite dimensional. Moreover the noise is allowed to be degenerate and the conditions required to apply Doob theorem are not always verified (see [7] for the theory of ergodicity when Doob Theorem can be applied).

The idea of compensating the degeneracy of the noise on some subspaces by dissipativity arguments has been introduced in [18, [19, and then in [3, 9]. In the same spirit, we consider systems which have only a finite number of unstable directions. In other words, the unstable manifold is finite-dimensional. Dissipative SPDEs such as the stochastic 2D Navier-Stokes (NS) and Complex GinzburgLandau (CGL) equations have this structure. The main requirement on the noise is that it is non degenerate in the unstable directions. Later, coupling methods have been introduced to prove exponential convergence to equilibrium (see [14, [21], 22], 23], 26] and [31]). 
Exponential Mixing for Stochastic PDEs: The Non-Additive Case.

These articles mainly deal with additive noises. Only in [26], the noise is allowed to have some dependence on the solution but it has to be of a very special form see below for more details. Moreover, the noise is assumed to be diagonal in the eigenbasis of the linear part of the equation.

In this article, we wish to get rid of these assumptions. This requires substantial adaptations in the method, for instance an auxiliary process is introduced. We develop a general ergodic criterion which ensures exponential mixing of the solution provided the image of the covariance operator of the noise contains the unstable modes.

Roughly speaking, our method allows to treat SPDEs perturbed by a noise of the type $\phi(u) d W$ where $u$ is the unknown of the equation and $W$ is the driving noise. Denoting by $P_{N}$ the projection onto the unstable modes, our main assumption is that the range of $\phi(u)$ contains the unstable modes $P_{N} H$. We think that this is a very natural condition. Note that with these notations, the above cited articles treat noises of the type $\phi d W, \phi$ being constant and diagonal and with the main assumption that the range of $\phi$ contains the unstable modes $P_{N} H$. In [26] (see also 30] $)$, the noise has the form $\phi\left(P_{N} u\right) d W$ with $\left(I-P_{N}\right) \phi\left(P_{N} u\right)=0$.

Our method is very general. Given a SPDE, it is sufficient to build an auxiliary process with good properties to apply our method and establish exponential convergence of the solutions to equilibrium. The technic to build this process depends on the type of SPDE. In fact, we distinguish three types of SPDEs. Examples of the two first types are given by NS and CGL. The third type of SPDE is more complicated to treat. It includes weakly damped but not strongly dissipative SPDEs. An example is the weakly damped Non-Linear Schrödinger (NLS) equation (see [8] for the case of an additive noise). We will study this equation in a forthcoming article.

The NS equations describe the time evolution of an incompressible fluid. It has been widely studied. Most of the articles cited above have been motivated by the application to this equation.

Originally introduced to describe a phase transition in superconductivity [12, the CGL equation also models the propagation of dispersive non-linear waves in various areas of physics such as hydrodynamics 28, 29], optics, plasma physics, chemical reaction [16]... The CGL equation arises in the same areas of physics as the nonlinear Schrödinger (NLS) equation. In fact, the CGL equation is obtained by adding two viscous terms to the NLS equation. The inviscid limits of the deterministic and stochastic CGL equation to the NLS equation are established in [2] and [24], respectively.

Ergodicity of the stochastic CGL equation is established in [1] when the noise is invertible and in [14] for the one-dimensional cubic case when the noise is diagonal, does not depend on the solution and is smooth in space. In 30, we have established exponential mixing of CGL driven by a noise which verifies the additional assumptions mentioned above under the $L^{2}$ or the $H^{1}$-subcritical conditions.

We hope that the method developed here can be combined with other recent ideas. For instance, in [15, 27, the case of NS perturbed by a four dimensional noise is treated. Hopefully, a four dimensional noise depending on the unknown could be studied. Another topic of interest is to try to prove exponential mixing in the three dimensional case for the transition semigroup constructed in [5]. This latter problem will be treated in a forthcoming paper. 
Exponential Mixing for Stochastic PDEs: The Non-Additive Case.

Another topic of interest is the study of more general noise. We will see in forthcoming papers that our Criterion (Theorem 2.1) could be extended to Levy type noises.

The remaining of the article is divided into four sections. First we define some preliminary definitions. In section 2, we prove our general criterion (Theorem 2.1) which states that a Markov process converges exponentially fast to equilibrium provided there exists an auxiliary process which verifies some properties. In section 3 and 4 we apply Theorem 3.4 to establish exponential mixing of the solutions of NS (Theorem 3.4) and CGL (Theorem 4.1).

\section{Preliminaries}

\subsection{Cylindrical Wiener process.}

Let $U, V, K$ be three separable Hilbert spaces. Here $\mathcal{L}(U ; K)\left(\operatorname{resp} \mathcal{L}_{2}(U ; K)\right)$ denotes the space of bounded (resp Hilbert-Schmidt) linear operators from the Hilbert space $U$ to $K$. For instance, the inclusion

$$
L^{2}\left((0,1)^{d}\right) \subset H^{-m}\left((0,1)^{d}\right)
$$

is Hilbert-Schmidt provided $m>\frac{d}{2}$.

The notion of cylindrical Wiener process is a generalization of the concept of Brownian motion and is used to model noise.

A process is said to be a cylindrical Wiener process of a Hilbert space $H$ if there exist an orthonormal basis $\left(e_{n}\right)_{n}$ of $H$, a family $\left(W_{n}\right)_{n}$ of independent brownian motions such that

$$
W=\sum_{n} W_{n} e_{n}
$$

It is important to notice that $W(t)$ is in $H$ with probability zero and that the sum converges almost surely (and for any moment) in $C((0, t) ; V)$ provided the inclusion $H \subset V$ is Hilbert-Schmidt. Moreover (1.2) true for a basis $\left(e_{n}\right)_{n}$ implies (1.2) for any orthonormal basis of $H$ (See [6] ).

Example 1.1. Let $d \in \mathbb{N}$. It is well-known that the inclusion $L^{2}\left((0,1)^{d}\right) \subset$ $H^{-m}\left((0,1)^{d}\right)$ is Hilbert-Schmidt provided $m>\frac{d}{2}$. So, a cylindrical Wiener process $W$ of $L^{2}\left((0,1)^{d}\right)$ is a continuous process of $H^{-m}\left((0,1)^{d}\right)$.

Remark 1.2. In the rest of this paper, when we will consider a Hilbert space $U$, we will implicitly fix a space $V$ such that $H \subset V$ is Hilbert-Schmidt. Then, all cylindrical Wiener processes on $U$ will be considered in the same space $V$. A random variable $X$ living in a space $E$ will be said to depend measurably on a cylindrical Wiener process $W$ on $U$ if there exists a map $f: C(0, \infty ; V) \rightarrow E$ such that $X=f(W)$.

\subsection{Topologies on the set of probability measures.}

Given a Polish space $E$, the space $\operatorname{Lip}_{b}(E)$ consists of all the bounded and Lipschitz real valued functions on $E$. Its norm is given by

$$
\|\varphi\|_{L}=|\varphi|_{\infty}+\underset{3}{L_{\varphi}}, \varphi \in \operatorname{Lip}_{b}(E)
$$


Exponential Mixing for Stochastic PDEs: The Non-Additive Case.

where $|\cdot|_{\infty}$ is the sup norm and $L_{\varphi}$ is the Lipschitz constant of $\varphi$. The space of probability measures on $E$ is denoted by $\mathcal{P}(E)$. It can be endowed with the norm defined by the total variation

$$
\|\mu\|_{\text {var }}=\sup \{|\mu(\Gamma)| \mid \Gamma \in \mathcal{B}(E)\},
$$

where we denote by $\mathcal{B}(E)$ the set of the Borelian subsets of $E$. It is well known that $\|\cdot\|_{v a r}$ is the dual norm of $|\cdot|_{\infty}$. We can also use the Wasserstein norm

$$
\|\mu\|_{*}=\sup _{\varphi \in L i p_{b}(E),\|\varphi\|_{L} \leq 1}\left|\int_{E} \varphi(u) d \mu(u)\right|,
$$

which is the dual norm of $\|\cdot\|_{L}$.

Remark 1.3. It is important to notice that the set of borelian subset of $\mathcal{P}(E)$ is the same for both the total variation and the Wasserstein norm.

Actually, a mapping $x \rightarrow \mathbb{P}_{x}$ taking value in $\mathcal{P}(E)$ is measurable if and only if the mapping $x \rightarrow \int_{E} f(y) d \mathbb{P}_{x}(y)$ is measurable for a suitable set of map $f: E \rightarrow \mathbb{R}$. For instance, the set of Lipschitz bounded map is such a suitable set.

In section 3 and 4 below, the measurability of the law of the process $u$ will be a consequence of the fact that

$$
\mathbb{E}\left(\left|u\left(t, W, u_{0}^{1}\right)-u\left(t, W, u_{0}^{2}\right)\right| \wedge 1\right) \rightarrow 0,
$$

provided $u_{0}^{2} \rightarrow u_{0}^{1}$.

\subsection{Couplings.}

We here recall some results about the coupling. Coupling is the basic key of the proof of our criterion (Theorem 2.1 below). But no known of coupling is required to apply this criterion (as we will see in section 3 and 4 ).

Let $\left(\Lambda_{1}, \Lambda_{2}\right)$ be two distributions on a two space $\left(E_{i}, \mathcal{E}_{i}\right)_{i=1,2}$. Let $(\Omega, \mathcal{F}, \mathbb{P})$ be a probability space and let $\left(Z_{1}, Z_{2}\right)$ be a couple random variables $(\Omega, \mathcal{F}) \rightarrow\left(E_{i}, \mathcal{E}_{i}\right)_{i=1,2}$. We say that $\left(Z_{1}, Z_{2}\right)$ is a coupling of $\left(\Lambda_{1}, \Lambda_{2}\right)$ if $\Lambda_{i}=\mathcal{D}\left(Z_{i}\right)$ for $i=1,2$. We have denoted by $\mathcal{D}\left(Z_{i}\right)$ the law of the random variable $Z_{i}$.

Let $\Lambda, \Lambda_{1}$ and $\Lambda_{2}$ be three probability measures on a same space $(E, \mathcal{E})$ such that $\Lambda_{1}$ and $\Lambda_{2}$ are absolutely continuous with respect to $\Lambda$. We set

$$
d\left(\Lambda_{1} \wedge \Lambda_{2}\right)=\left(\frac{d \Lambda_{1}}{d \Lambda} \wedge \frac{d \Lambda_{2}}{d \Lambda}\right) d \Lambda
$$

This definition does not depend on the choice of $\Lambda$ and we have

$$
\left\|\Lambda_{1}-\Lambda_{2}\right\|_{v a r}=\frac{1}{2} \int_{E}\left|\frac{d \Lambda_{1}}{d \Lambda}-\frac{d \Lambda_{2}}{d \Lambda}\right| d \Lambda .
$$

Next result is a fundamental result in the coupling methods (See for instance 25]).

Lemma 1.4. Let $\left(\Lambda_{1}, \Lambda_{2}\right)$ be two probability measures on a same $(E, \mathcal{E})$. Then

$$
\left\|\Lambda_{1}-\Lambda_{2}\right\|_{v a r}=\min \mathbb{P}\left(Z_{1} \neq Z_{2}\right) .
$$

The minimum is taken over all couplings $\left(Z_{1}, Z_{2}\right)$ of $\left(\Lambda_{1}, \Lambda_{2}\right)$. There exists a coupling which reaches the minimum value. It is called a maximal coupling and has the following property:

$$
\mathbb{P}\left(Z_{1}=Z_{2}, Z_{1} \in \Gamma\right)=\left(\Lambda_{1} \wedge \Lambda_{2}\right)(\Gamma) \text { for any } \Gamma \in \mathcal{E} .
$$


Exponential Mixing for Stochastic PDEs: The Non-Additive Case.

It is interesting to remark that if $\Lambda_{1}$ is absolutely continuous with respect to $\Lambda_{2}$, we have

$$
\left\|\Lambda_{1}-\Lambda_{2}\right\|_{v a r} \leq \frac{1}{2} \sqrt{\int\left(\frac{d \Lambda_{1}}{d \Lambda_{2}}\right)^{2} d \Lambda_{2}-1} .
$$

In order to estimate the bound given in Lemma 1.4 we use either (1.3) or the following result which is lemma D.1 of [26] and which is very useful in order to bound below the probability that a maximal coupling get coupled.

Lemma 1.5. Let $\Lambda_{1}$ and $\Lambda_{2}$ be two equivalent probability measures on a space $(E, \mathcal{E})$. Then for any $p>1$ and any event $A$ of $E$

$$
I_{p}=\int_{A}\left(\frac{d \Lambda_{1}}{d \Lambda_{2}}\right)^{p} d \Lambda_{1}<\infty \quad \text { implies } \quad\left(\Lambda_{1} \wedge \Lambda_{2}\right)(A) \geq\left(1-\frac{1}{p}\right)\left(\frac{\Lambda_{1}(A)^{p}}{p I_{p}}\right)^{\frac{1}{p-1}} .
$$

Next result is a refinement of Lemma 1.4 used in [26].

Proposition 1.6. Let $E$ and $F$ be two Polish spaces, $f_{0}: E \rightarrow F$ be a measurable map and $\left(\Lambda_{1}, \Lambda_{2}\right)$ be two probability measures on $E$. We set

$$
\lambda_{i}=f_{0}^{*} \Lambda_{i}, \quad i=1,2 .
$$

Then there exists a coupling $\left(V_{1}, V_{2}\right)$ of $\left(\Lambda_{1}, \Lambda_{2}\right)$ such that $\left(f_{0}\left(V_{1}\right), f_{0}\left(V_{2}\right)\right)$ is a maximal coupling of $\left(\lambda_{1}, \lambda_{2}\right)$.

Setting $f_{0}:(u, v) \rightarrow u, V_{1}=\left(U_{1}, \widetilde{U}\right)$ and $V_{2}=\left(U_{2}, U_{2}\right)$, it follows.

Corollary 1.7. Let $E$ be a Polish space, $(\Omega, \mathcal{F}, \mathbb{P})$ be a probability space and $\left(U_{1}, U_{2}, \widetilde{U}\right)$ be three random variables on $(\Omega, \mathcal{F}, \mathbb{P})$ taking value in $E$.

Then there exists a triplet $\left(u_{1}, u_{2}, \widetilde{u}\right)$ such that $\left(u_{2}, \widetilde{u}\right)$ is a maximal coupling of $\left(\mathcal{D}\left(U_{2}\right), \mathcal{D}(\widetilde{U})\right)$ and such that the law of $\left(u_{1}, \widetilde{u}\right)$ is $\mathcal{D}\left(U_{1}, \widetilde{U}\right)$.

Remark 1.8 (Measurability and Markov property).

Let $\left(u_{0}^{1}, u_{0}^{2}\right) \rightarrow\left(U\left(u_{0}^{1}\right), \widetilde{U}\left(u_{0}^{1}, u_{0}^{2}\right)\right)$ such that $\left(u_{0}^{1}, u_{0}^{2}\right) \rightarrow \mathcal{D}\left(U\left(u_{0}^{1}\right), \widetilde{U}\left(u_{0}^{1}, u_{0}^{2}\right)\right)$ is measurable (See Remark [1.3). It is possible to build the triplet $\left(u_{1}, u_{2}, \widetilde{u}\right)$ of Corollary 1.7 associated to $\left(U\left(u_{0}^{1}\right), U\left(u_{0}^{2}\right), \widetilde{U}\left(u_{0}^{1}, u_{0}^{2}\right)\right)$ such that $\left(u_{0}^{1}, u_{0}^{2}\right) \rightarrow\left(u_{1}, u_{2}, \widetilde{u}\right)$ is measurable (See for instance Remark A.1 of [30]).

Now, assume that $u_{0}^{1}, u_{0}^{2}, u_{1}, u_{2}$ live in the same space and let $\left(\left(u_{0}^{1}, u_{0}^{2}\right) \rightarrow\left(u_{1}^{n}, u_{2}^{n}, \widetilde{u}^{n}\right)\left(u_{0}^{1}, u_{0}^{2}\right)\right)_{n}$ be a sequence of independent versions of the triplet $\left(u_{0}^{1}, u_{0}^{2}\right) \rightarrow\left(u_{1}, u_{2}, \widetilde{u}\right)\left(u_{0}^{1}, u_{0}^{2}\right)$. Since it measurably depends on $\left(u_{0}^{1}, u_{0}^{2}\right)$, we can iterate this sequence, i.e. we set

$$
\left\{\begin{aligned}
\left(u_{1}(0), u_{2}(0)\right) & =\left(u_{0}^{1}, u_{0}^{2}\right), \\
\left(u_{1}(n+1), u_{2}(n+1), \widetilde{u}(n+1)\right) & =\left(u_{1}^{n+1}, u_{2}^{n+1}, \widetilde{u}^{n+1}\right)\left(u_{1}(n), u_{2}(n)\right) .
\end{aligned}\right.
$$

It easily follows that $\left(\left(u_{1}(n), u_{2}(n), \widetilde{u}(n)\right)\right)_{n}$ is a Markov chain.

Another viewpoint of what we have done is the following. We first have set

$$
\left(u_{1}(0), u_{2}(0)\right)=\left(u_{0}^{1}, u_{0}^{2}\right) .
$$

Then, assuming that $\left(u_{1}, u_{2}, \widetilde{u}\right)$ is build on $\{0 \ldots, n\}$, we have fixed a path and we have build $\left(u_{1}(n+1), u_{2}(n+1), \widetilde{u}(n+1)\right)$ as a triplet of $\left(U\left(u_{1}(n)\right), U\left(u_{2}(n)\right), \widetilde{U}\left(u_{1}(n), u_{2}(n)\right)\right)$. Finally, we have integrated the probability over the path on $\{0, \ldots, n\}$. This is this viewpoint we will use in the next sections. 
Exponential Mixing for Stochastic PDEs: The Non-Additive Case.

\section{A GENERAL CRITERION}

This section is devoted to the statement and the proof of a general criterion -Theorem [2.1 which ensures exponential mixing of a Markov process $u$, provided there exists an auxiliary process $\widetilde{u}$ which verifies some properties. In particular, Theorem 3.4 below (resp Theorem 4.1) that states that the solutions of NS (resp CGL) are exponentially mixing is a Corollary of Theorem 2.1

\subsection{Statement of the criterion.}

Let $\left(U,|\cdot|_{U}\right)$ be a Hilbert space and $W$ be a cylindrical Wiener process on $U$. We are concerned with a continuous homogenous weak Markov process $u$ taking value in a Polish space $\left(H, d_{H}\right)$. This Markov process $u$ is assumed to be a non anticipative measurable map of $W$ (See Remark [1.2). Since $u$ is a Markov process, it is assumed that its law $\mathcal{D}(u)$ is measurably depending of its initial condition $u_{0}$ (See Remark 1.3). We will denote the dependance of $u$ with respect to $\left(t, W, u_{0}\right)$ as follows

$$
u(t)=u\left(t, W, u_{0}\right) .
$$

We denote by $\left(\mathcal{P}_{t}\right)_{t \in \mathbb{R}^{+}}$the Markov transition semi-group associated to the Markov family $\left(u\left(\cdot, W, u_{0}\right)\right)_{u_{0} \in H}$.

We first assume the existence of an auxiliary process $\widetilde{u}$ such that $(u, \widetilde{u})$ is Markov. A0 There exists a continuous process $\widetilde{u}$ taking value in $H$ and that is a non anticipative measurable map of $W$. Moreover $(u, \widetilde{u})$ is a homogenous weak Markov process and its law $\mathcal{D}(u, \widetilde{u})$ is measurably depending of its initial condition $\left(u_{0}, \widetilde{u}_{0}\right)$.

We will denote the dependance of $(u, \widetilde{u})$ with respect to $\left(t, W, u_{0}, \widetilde{u}_{0}\right)$ as follows

$$
(u(t), \widetilde{u}(t))=\left(u\left(t, W, u_{0}\right), \widetilde{u}\left(t, W, u_{0}, \widetilde{u}_{0}\right)\right) .
$$

The next assumptions involve a positive measurable functional $\mathcal{H}: H \rightarrow \mathbb{R}^{+}$, which plays the role of a Lyapunov functional. We assume that there exist $\gamma, C_{1}, C>$ 0 and a mapping $h: H^{2} \rightarrow U$ such that the following hold.

A1 There exists a family $\left(C_{\alpha}^{\prime}\right)_{\alpha \in(0, \infty)}$, such that for any $u_{0} \in H$, any $t \geq 0$, any $\alpha>0$ and any stopping time $\tau \geq 0$,

$$
\left\{\begin{aligned}
\mathbb{E}\left(\mathcal{H}\left(u\left(t, W, u_{0}\right)\right)\right) & \leq e^{-\gamma t} \mathcal{H}\left(u_{0}\right)+C_{1}, \\
\mathbb{E}\left(e^{-\alpha \tau} \mathcal{H}\left(u\left(\tau, W, u_{0}\right)\right) 1_{\tau<\infty}\right) & \leq \mathcal{H}\left(u_{0}\right)+C_{\alpha}^{\prime} .
\end{aligned}\right.
$$

A2 For any $\left(u_{0}^{1}, u_{0}^{2}\right) \in H^{2}$, for any couple $\left(W_{1}, W_{2}\right)$ of cylindrical Wiener processes of $U$ and for any $t \geq 0$, we have

$$
\mathbb{P}\left(d_{H}\left(u_{1}(t), u_{2}(t)\right) \geq C e^{-\gamma t} \text { and } \widetilde{u}=u_{2} \text { on }[0, t]\right) \leq C e^{-\gamma t},
$$

where

$$
\left\{\begin{aligned}
u_{i}(t) & =u\left(t, W_{i}, u_{0}^{i}\right) \quad \text { for } \quad i=1,2 \\
\widetilde{u}(t) & =\widetilde{u}\left(t, W_{1}, u_{0}^{1}, u_{0}^{2}\right) \\
2 C_{1} & \geq \mathcal{H}\left(u_{0}^{1}\right)+\mathcal{H}\left(u_{0}^{2}\right) .
\end{aligned}\right.
$$

A3 For any $\left(t, u_{0}^{1}, u_{0}^{2}\right) \in(0, \infty) \times H^{2}$, we have almost surely

$$
\widetilde{u}\left(t, W, u_{0}^{1}, u_{0}^{2}\right)=u\left(t, W+\int_{0}^{\cdot} h\left(u\left(s, W, u_{0}^{1}\right), \widetilde{u}\left(s, W, u_{0}^{1}, u_{0}^{2}\right)\right) d s, u_{0}^{2}\right) .
$$


Exponential Mixing for Stochastic PDEs: The Non-Additive Case.

A4 For any couple $\left(W_{1}, W_{2}\right)$ of cylindrical Wiener processes of $U$ and for any $\left(t_{0}, u_{0}^{1}, u_{0}^{2}\right) \in[0, \infty) \times H^{2}$

$$
\mathbb{P}\left(\int_{t_{0}}^{\tau}|h(t)|_{U}^{2} d t \geq C e^{-\gamma t_{0}} \text { and } \widetilde{u}=u_{2} \text { on }[s, \tau]\right) \leq C e^{-\gamma t_{0}},
$$

where $\left(\widetilde{u}, u_{2}\right)$ are defined in $\mathbf{A 2}$, where $\tau \geq t_{0}$ is any stopping time and where

$$
\left\{\begin{aligned}
h(t) & =h\left(u\left(t, W, u_{0}^{1}\right), \widetilde{u}\left(t, W, u_{0}^{1}, u_{0}^{2}\right)\right), \\
2 C_{1} & \geq \mathcal{H}\left(u_{0}^{1}\right)+\mathcal{H}\left(u_{0}^{2}\right) .
\end{aligned}\right.
$$

A5 There exists $p_{1}>0$ such that for any $\left(u_{0}^{1}, u_{0}^{2}\right) \in H^{2}$, we have

$$
\mathbb{P}\left(\int_{0}^{\infty}|h(t)|_{U}^{2} d t \leq C\right) \geq p_{1},
$$

where

$$
\left\{\begin{aligned}
h(t) & =h\left(u\left(t, W, u_{0}^{1}\right), \widetilde{u}\left(t, W, u_{0}^{1}, u_{0}^{2}\right)\right) \\
2 C_{1} & \geq \mathcal{H}\left(u_{0}^{1}\right)+\mathcal{H}\left(u_{0}^{2}\right) .
\end{aligned}\right.
$$

We now state our criterion.

Theorem 2.1. Under the above assumptions, there exists a unique stationary probability measure $\mu$ of $\left(\mathcal{P}_{t}\right)_{t \in \mathbb{R}^{+}}$on $H$. Moreover, $\mu$ satisfies

$$
\int_{H} \mathcal{H}(u) d \mu(u)<\infty,
$$

and there exist $C, \gamma^{\prime}>0$ such that for any $\lambda \in \mathcal{P}(H)$

$$
\left\|\mathcal{P}_{t}^{*} \lambda-\mu\right\|_{*} \leq C e^{-\gamma^{\prime} t}\left(1+\int_{H} \mathcal{H}(u) d \lambda(u)\right) .
$$

Theorem 2.1 is proved in sections $2.2, \ldots, 2.9$ hereafter. Let us quickly sketch the proof.

Assumption $\mathbf{A 1}$ is standard and ensures that 2.1) holds and that the time of return of the process in any ball of radius greater than $2 C_{1}$ admits an exponential moment. Assumption A2 states that $u\left(t, W, u_{0}^{1}\right)$ and $\widetilde{u}\left(t, W, u_{0}^{1}, u_{0}^{2}\right)$ become close exponentially fast in probability.

Assumption $\mathbf{A} 3$ means that the law $\widetilde{u}\left(\cdot, W, u_{0}^{1}, u_{0}^{2}\right)$ is the law of $u\left(\cdot, W^{\prime}, u_{0}^{2}\right)$ where $W^{\prime}$ is a drifted Wiener process. Assumptions A4 and A5 imply that the Novikov condition holds for a truncation of $\widetilde{u}$. So combining A3, A4 and A5, a Girsanov Transform can be used to build a couple of Wiener processes $\left(W_{1}, W_{2}\right)$ such that

$$
\widetilde{u}\left(\cdot, W_{1}, u_{0}^{1}, u_{0}^{2}\right)=u\left(\cdot, W_{2}, u_{0}^{2}\right),
$$

with a positive probability.

Conclusion: Iterating and combining the three properties, we can conclude by remarking that it allows to control the probability of $u\left(t, W_{1}, u_{0}^{1}\right)$ and $u\left(t, W_{2}, u_{0}^{2}\right)$ being very close. Actually, we wait for entering the ball of radius $2 C_{1}$. Then, the probability that $\left(\widetilde{u}\left(\cdot-s, W_{1}, u_{1}(s), u_{2}(s)\right), u\left(\cdot-s, W_{2}, u_{2}(s)\right)\right)$ get coupled for any time is bounded below. If it fails to couple, we wait again for entering the ball 
Exponential Mixing for Stochastic PDEs: The Non-Additive Case.

of radius $2 C_{1}$ and we retry. It follows that there exists a random time $T_{*}$ with exponential moment such that

$$
\widetilde{u}\left(\cdot-T_{*}, W_{1}, u_{1}\left(T_{*}\right), u_{s}^{2}\right)=u\left(\cdot-T_{*}, W_{2}, u_{2}\left(T_{*}\right)\right) .
$$

So applying A2, we are able to conclude.

Remark 2.2. For the Navier-Stokes and the Complex Ginzburg-Landau equations treated in section 3 and 4 , we use the same functional $\mathcal{H}=|\cdot|^{2}$. More complicated choices may be necessary as in the case of the weakly damped Non Linear Schrödinger equation treated in [8].

Remark 2.3 (Finest assumption). It is possible to refine our criterion as follows. In our proof and especially in section [2.5. assumption $\mathbf{A 5}$ is used to prove the following irreducibility argument

A6 For any $r_{0}>0$, there exist $T, p_{0}>0$ and a measurable family of coupling $\left(u_{0}^{1}, u_{0}^{2}\right) \rightarrow\left(u_{1}, u_{2}\right)$ of $\left(\mathcal{D}\left(u\left(\cdot, W, u_{0}^{1}\right), u\left(\cdot, W, u_{0}^{2}\right)\right)\right)$ such that

$$
\mathbb{P}\left(d_{H}\left(u_{1}(T), u_{2}(T)\right) \leq r_{0}\right) \geq p_{0},
$$

provided

$$
2 C_{1} \geq \mathcal{H}\left(u_{0}^{1}\right)+\mathcal{H}\left(u_{0}^{2}\right) .
$$

Actually A0,.., A4 and A6 are sufficient to prove (2.1) and (2.2) provided the constant $C$ in our assumptions verifies suitable conditions when $d_{H}\left(u_{0}^{1}, u_{0}^{2}\right) \leq r_{0}$. For NS and CGL, it is easier to prove directly $\mathbf{A 0}, \ldots, \mathbf{A 5}$. But, for some equation, $\mathbf{A 5}$ is not true and $\mathbf{A 6}$ can easily be proved. In that case, the criterion should be adapted.

Remark 2.4. It we replace the term $e^{-\gamma t}$ by a negative power of $t$ in our assumptions, we obtain a Theorem analogous to Theorem [2.1 but where convergence is polynomial instead of being exponential.

We have seen in 8 that for the stochastic Non-Linear Schrödinger (NLS) equation the control of the energy is polynomial. Hence, we think that the polynomial version of our criterion is the good framework when studying weakly damped SPDE.

Moreover we will see in Remarks 3.8 and 4.7 that there exist some variations of Theorem 3.4 and 4.1] whose convergence is polynomial. To establish such results, we need the polynomial version of Theorem 2.1.

Remark 2.5 (Levy type noise). It is possible to obtain a Theorem analogous to Theorem 2.1 where the noise $W$ is replaced by a Levy process $N(d t d z)$ (or by a couple $(W, N(d t d z)))$. But the proof is more complicated because the Girsanov transform for measure causes some problems.

This generalization is required by some SPDEs that naturally appear in physical problems such as the stochastic Non-Linear Schrödinger (NLS) driven by LevyBrown noise. These results would be established in forthcoming papers.

The remaining of this section is devoted to the proof of Theorem 2.1.

\subsection{Building of a coupling of the solutions.}

Let us denote by $\left(u_{0}^{1}, u_{0}^{2}\right)$ two initial conditions in $H$ and by $T$ a positive real number. 
Exponential Mixing for Stochastic PDEs: The Non-Additive Case.

Applying Corollary 1.7 we build a family of independent measurable map (See Remark 1.8

$$
\left(\left(u_{0}^{1}, u_{0}^{2}\right) \rightarrow\left(u_{1}^{n}, u_{2}^{n}, \widetilde{u}^{n}\right)\left(u_{0}^{1}, u_{0}^{2}\right)\right)_{n \in \mathbb{N}},
$$

such that the law of $\left(u_{1}^{n}, \widetilde{u}^{n}\right)\left(u_{0}^{1}, u_{0}^{2}\right)$ is $\left(\mathcal{D}\left(u\left(\cdot, W, u_{0}^{1}\right)\right), \mathcal{D}\left(\widetilde{u}\left(\cdot, W, u_{0}^{1}, u_{0}^{2}\right)\right)\right)$ and $\left(u_{2}^{n}, \widetilde{u}^{n}\right)\left(u_{0}^{1}, u_{0}^{2}\right)$ is a maximal coupling of $\left(\mathcal{D}\left(u\left(\cdot, W, u_{0}^{2}\right)\right), \mathcal{D}\left(\widetilde{u}\left(\cdot, W, u_{0}^{1}, u_{0}^{2}\right)\right)\right)$ on $(0, T]$.

We now build the coupling $\left(u_{1}, u_{2}\right)$ of $\left(\mathcal{D}\left(u\left(\cdot, W, u_{0}^{1}\right)\right), \mathcal{D}\left(u\left(\cdot, W, u_{0}^{2}\right)\right)\right)$ on $[0, n T]$ by induction on $n \in \mathbb{N}$. Indeed we first set

$$
u_{i}(0)=u_{0}^{i}, \quad i=1,2 .
$$

Assume that $\left(u_{1}, u_{2}, \widetilde{u}\right)$ is build on $[0, n T]$, we extend it on $[0,(n+1) T]$ be setting

$$
\left(u_{1}, u_{2}, \widetilde{u}\right)(n T+\cdot)=\left(u_{1}^{n}, u_{2}^{n}, \widetilde{u}^{n}\right)\left(u_{1}(n T), u_{2}(n T)\right) .
$$

It easily follows that $\left(u_{1}, u_{2}\right)$ is a coupling of $\left(\mathcal{D}\left(u\left(\cdot, W, u_{0}^{1}\right)\right), \mathcal{D}\left(u\left(\cdot, W, u_{0}^{2}\right)\right)\right)$ on $(0, \infty)$ and that the triplet $\left(u_{1}, u_{2}, \widetilde{u}\right)$ is homogenous weak Markov at discrete time $T \mathbb{N}$. Since since the strong Markov property is equivalent to the weak Markov property when working at discrete times, it means that for any stopping times $\tau \in T \mathbb{N} \cup\{\infty\}$

$$
\mathbb{E}_{u_{0}^{1}, u_{0}^{2}}\left(1_{\tau<\infty} f\left(\left(u_{1}, u_{2}, \widetilde{u}\right)\right) o \theta_{\tau} \mid \mathcal{F}_{\tau}\right)=1_{\tau<\infty} \mathbb{E}_{u_{1}(\tau), u_{2}(\tau)}\left(f\left(u_{1}, u_{2}, \widetilde{u}\right)\right),
$$

where we have denoted by $\left(\theta_{t}\right)_{t}$ the family of shift operators

$$
\left(f\left(u_{1}, u_{2}, \widetilde{u}\right)\right) o \theta_{t}=f\left(u_{1}(t+\cdot), u_{2}(t+\cdot), \widetilde{u}(t+\cdot)\right) .
$$

\subsection{Introduction of $l_{0}$.}

In order to apply $\mathbf{A 2}$, we define a family of integer valued random process $\left(l_{0}(k)\right)_{k \in \mathbb{N} \cup\{\infty\}}$ which is particularly convenient when deriving properties of the triplet

$$
l_{0}(k)=\min \left\{l \leq k \mid P_{l, k}\right\},
$$

where $\min \phi=\infty$ and

$$
\left(P_{l, k}\right)\left\{\begin{array}{l}
\widetilde{u}=u_{2} \text { on }(l T, k T), \\
\mathcal{H}\left(u_{1}(l T)\right)+\mathcal{H}\left(u_{2}(l T)\right) \leq 2 C_{1} .
\end{array}\right.
$$

The interest of $l_{0}$ comes from the fact that it allows to apply $\mathbf{A 2}$ that can be rewritten in the following form

$$
\mathbb{E}\left(\left|u_{2}(t)-u_{1}(t)\right| \wedge 1_{l_{0}(\infty) \leq l}\right) \leq C e^{-\gamma(t-l T)},
$$

provided $t \geq l T$.

\subsection{Construction of a useful coupling.}

In subsections 2.5 and 2.6] we are interested with the law of $\left(u_{1}, u_{2}, \widetilde{u}\right)$ conditioned by $l_{0}(k)=0$. For that purpose we fix a path of $\left(u_{1}, u_{2}, \widetilde{u}\right)$ on $[0, k T]$ such that $l_{0}(k)=0$ and we build $\left(u_{1}, u_{2}, \widetilde{u}\right)$ on $(k T,(k+1) T]$ as in subsection 2.2 by applying Corollary [1.7 When the path of $\left(u_{1}, u_{2}, \widetilde{u}\right)$ is fixed on $[0, k T]$, the probability is denoted by $\mathbb{Q}$.

To understand well the link between $\mathbb{P}$ defined in section 2.2 and $\mathbb{Q}$, notice that

$$
\mathbb{Q}\left(\left(u_{1}, u_{2}, \widetilde{u}\right) \in B\right)=\mathbb{P}\left(\left(u_{1}, u_{2}, \widetilde{u}\right) \in B \mid \mathcal{F}_{k T}\right),
$$

provided $B$ is a measurable subset of $C(k T,(k+1) T ; H)^{3}$. 
It is not easy to work directly with the couple $\left(\widetilde{u}, u_{2}\right)$. We prefer working with cylindrical Wiener processes because of a very useful tool, namely the Girsanov transform. That is the reason why we build the following coupling.

We first set

$$
\left\{\begin{aligned}
h(t, W) & =h\left(u\left(t-k T, W, u_{1}(k T)\right), \widetilde{u}\left(t-k T, W, u_{1}(k T), u_{2}(k T)\right)\right) \\
\tau(W) & =\inf \left\{\left.t \in(k T,(k+1) T)\left|\int_{k T}^{t}\right| h(t, W)\right|^{2} d t>2 C e^{-\gamma k T}\right\}
\end{aligned}\right.
$$

Then, applying Corollary 1.7 to

$$
\left(W, W, W+\int_{k T}^{\tau(W) \wedge \cdot} h(t, W) d t\right)
$$

we obtain a couple $\left(W_{1}, W_{2}\right)$ of cylindrical Wiener processes such that

$$
\left(W_{2}, W_{1}+\int_{k T}^{\tau\left(W_{1}\right) \wedge \cdot} h\left(t, W_{1}\right) d t\right),
$$

is a maximal coupling on $[k T,(k+1) T]$.

Since $l_{0}(k)=0$, it follows from the definition of $l_{0}(k+1)$ that

$$
\mathbb{Q}\left(l_{0}(k+1)=0\right)=\mathbb{Q}\left(\widetilde{u}=u_{2}\right) .
$$

Applying the maximal coupling property of the couple $\left(\widetilde{u}, u_{2}\right)$, it follows from the fact that $\left(\widetilde{u}\left(\cdot-k T, W_{1}, u_{1}(k T), u_{2}(k T)\right), u\left(\cdot-k T, W_{2}, u_{2}(k T)\right)\right)$ is a coupling of $\left(\mathcal{D}(\widetilde{u}), \mathcal{D}\left(u_{2}\right)\right)$ on $(k T,(k+1) T)$

$$
\mathbb{Q}\left(l_{0}(k+1)=0\right) \geq \mathbb{Q}\left(\widetilde{u}\left(\cdot-k T, W_{1}, u_{1}(k T), u_{2}(k T)\right)=u\left(\cdot-k T, W_{2}, u_{2}(k T)\right)\right),
$$

which yields, by $\mathbf{A} \mathbf{3}$,

$$
\mathbb{Q}\left(l_{0}(k+1)=0\right) \geq \mathbb{Q}\left(W_{2}=W_{1}+\int_{k T}^{\tau\left(W_{1}\right) \wedge \cdot} h\left(t, W_{1}\right) d t \text { and } \tau\left(W_{1}\right)=(k+1) T\right) .
$$

Let us set

$$
\left\{\begin{aligned}
A & =\{W \mid \tau(W)=(k+1) T\}, \\
\Lambda_{1} & =\mathcal{D}(W), \\
\Lambda_{2} & =\mathcal{D}\left(W+\int_{k T}^{\tau(W) \wedge \cdot} h(t, W) d t\right) .
\end{aligned}\right.
$$

Novikov condition is obviously verified. So, Girsanov Transform gives

$$
\left(\frac{d \Lambda_{1}}{d \Lambda_{2}}\right)(W)=\exp \left(\int_{k T}^{\tau(W)} h(t, W) d W(t)-\frac{1}{2} \int_{k T}^{\tau(W)}|h(t, W)|^{2} d t\right),
$$

which yields

$$
\int\left(\frac{d \Lambda_{1}}{d \Lambda_{2}}\right)^{2} d \Lambda_{1} \leq \mathbb{E}\left(e^{\int_{k T}^{\tau(W)}|h(t, W)|^{2} d t}\right) \leq e^{2 C e^{-\gamma k T}}
$$




\subsection{Probability that $l_{0}(1)=0$.}

In that subsection, we treat the case $k=0$, i.e. we assume that $l_{0}(0)=0$. In that case $\mathbb{P}=\mathbb{Q}$.

Applying the maximal coupling property of the couple $\left(W_{2}, W_{1}+\int_{0}^{\tau\left(W_{1}\right) \wedge \cdot} h\left(t, W_{1}\right) d t\right)$, it follows from (2.6) and from Lemmas 1.4 and 1.5 that

$$
\mathbb{P}\left(l_{0}(1)=0\right) \geq \frac{1}{4}\left(\int\left(\frac{d \Lambda_{1}}{d \Lambda_{2}}\right)^{2} d \Lambda_{1}\right)^{-1} \Lambda_{1}(A) .
$$

It follows from A5 that

$$
\Lambda_{1}(A) \geq p_{1}
$$

Combining (2.8) and (2.7), we obtain

$$
\mathbb{P}\left(l_{0}(1)=0\right) \geq \frac{p_{1}}{4} e^{-2 C} .
$$

2.6. Probability that $l_{0}(k+1)=0$.

Recall that, for the probability $\mathbb{Q}$, we have fixed a path of $\left(u_{1}, u_{2}, \widetilde{u}\right)$ on $[0, k T]$ such that $l_{0}(k)=0$ and we have build $\left(u_{1}, u_{2}, \widetilde{u}\right)$ on $[k T,(k+1) T]$ as in subsection 2.2

Notice that (2.6) can be rewritten as follows

$$
\mathbb{Q}\left(l_{0}(k+1) \neq 0\right) \leq \mathbb{Q}\left(W_{2} \neq W_{1}+\int_{k T}^{\tau\left(W_{1}\right) \wedge \cdot} h\left(t, W_{1}\right) d t \text { or } \tau\left(W_{1}\right)<(k+1) T\right),
$$

which yields

$$
\begin{array}{r}
\mathbb{Q}\left(l_{0}(k+1) \neq 0\right) \leq \mathbb{Q}\left(W_{2}=W_{1}+\int_{k T}^{\tau\left(W_{1}\right) \wedge \cdot} h\left(t, W_{1}\right) d t \text { and } \tau\left(W_{1}\right)<(k+1) T\right) \\
+\mathbb{Q}\left(W_{2} \neq W_{1}+\int_{k T}^{\tau\left(W_{1}\right) \wedge \cdot} h\left(t, W_{1}\right) d t\right) .
\end{array}
$$

Notice that, by A3,

$$
\begin{gathered}
\mathbb{Q}\left(W_{2}=W_{1}+\int_{k T}^{\tau\left(W_{1}\right) \wedge \cdot} h\left(t, W_{1}\right) d t \text { and } \tau\left(W_{1}\right)<(k+1) T\right) \leq \\
\mathbb{Q}\left(u\left(\cdot-k T, W_{2}, u_{2}(k T)\right)=\widetilde{u}\left(\cdot-k T, W_{1}, u_{1}(k T), u_{2}(k T)\right) \text { and } \tau\left(W_{1}\right)<(k+1) T\right) .
\end{gathered}
$$

Applying the maximal coupling property of the couple $\left(W_{2}, W_{1}+\int_{0}^{\tau\left(W_{1}\right) \wedge \cdot} h\left(t, W_{1}\right) d t\right)$, it follows from Lemma 1.4 and from (1.3), (2.7) that

$$
\mathbb{Q}\left(W_{2} \neq W_{1}+\int_{k T}^{\tau\left(W_{1}\right) \wedge} h\left(t, W_{1}\right) d t\right) \leq C e^{-\gamma k T} .
$$

Combining (2.10), (2.11), (2.12), we obtain

$$
\begin{aligned}
& \mathbb{Q}\left(l_{0}(k+1) \neq 0\right) \leq C e^{-\gamma k T}+ \\
& \mathbb{Q}\left(u\left(\cdot-k T, W_{2}, u_{2}(k T)\right)=\widetilde{u}\left(\cdot-k T, W_{1}, u_{1}(k T), u_{2}(k T)\right) \text { and } \tau\left(W_{1}\right)<(k+1) T\right) .
\end{aligned}
$$

Notice that, since we have fixed $\left(u_{1}, u_{2}, \widetilde{u}\right)$ on $[0, k T]$, we cannot apply $\mathbf{A} 4$ to bound the right hand side. 
Exponential Mixing for Stochastic PDEs: The Non-Additive Case.

So using (2.4) and integrating (2.13) by $\left.\left(u_{1}, u_{2}, \widetilde{u}\right)\right|_{[0, k T]}$ over $l_{0}(k)=0$, we deduce from $\mathbf{A} 4$ that

$$
\mathbb{P}\left(l_{0}(k+1) \neq 0 \text { and } l_{0}(k)=0\right) \leq C e^{-\gamma k T} .
$$

2.7. Probability that $l_{0}(\infty)=0$ and time of failure.

We first assume that $l_{0}(0)=0$.

Notice that, since $l_{0}(k)=0$ implies $l_{0}(l)=0$ for any $\infty \geq k \geq l \geq 0$, it follows that

$$
\mathbb{P}\left(l_{0}(\infty) \neq 0\right) \leq \sum_{k=0}^{\infty} \mathbb{P}\left(l_{0}(k+1) \neq 0 \text { and } l_{0}(k)=0\right) .
$$

Combining (2.9) and (2.14), we obtain

$$
\mathbb{P}\left(l_{0}(\infty) \neq 0\right) \leq 1-\frac{p_{1}}{4} e^{-2 C}+\frac{e^{-\gamma T}}{1-e^{-\gamma T}} C .
$$

It follows that there exists $T_{0}$ such that $T \geq T_{0}$ implies

$$
\mathbb{P}\left(l_{0}(\infty)=0\right) \geq p_{0}=\frac{p_{1}}{8} e^{-2 C} .
$$

We now fix $T=T_{0}$.

We denote by $\sigma$ the time where $l_{0}$ stop being zero, i.e.

$$
\sigma=\inf \left\{n \in \mathbb{N} \mid l_{0}(n)>0\right\} .
$$

It follows from (2.14) that

$$
\mathbb{P}(\sigma=k+1) \leq C e^{-\gamma k T},
$$

which yields that, for any $\alpha \in\left(0, \frac{\gamma T}{6}\right)$,

$$
\mathbb{E}\left(e^{3 \alpha \sigma} 1_{\sigma<\infty}\right) \leq c .
$$

2.8. Moment of $l_{0}(\infty)$.

From now, we stop assuming that $l_{0}(0)=0$.

Notice that it follows from A1 that the time of return $\delta$ in the ball of radius $2 C_{1}$ admits an exponential moment, i.e., there exist $\alpha \in\left(0, \frac{\gamma T}{6}\right)$ and $c>0$ such that

$$
\mathbb{E}\left(e^{\alpha \delta}\right) \leq c \sqrt{1+\mathcal{H}\left(u_{0}^{1}\right)+\mathcal{H}\left(u_{0}^{2}\right)},
$$

where

$$
\delta=\min \left\{n \in \mathbb{N} \mid \mathcal{H}\left(u_{1}(n T)\right)+\mathcal{H}\left(u_{2}(n T)\right) \leq 2 C_{1}\right\} .
$$

For a proof, see for instance (1.56) of [30] and apply a Schwarz inequality.

Now we combine $\delta$ and $\sigma$ and we iterate them. We set

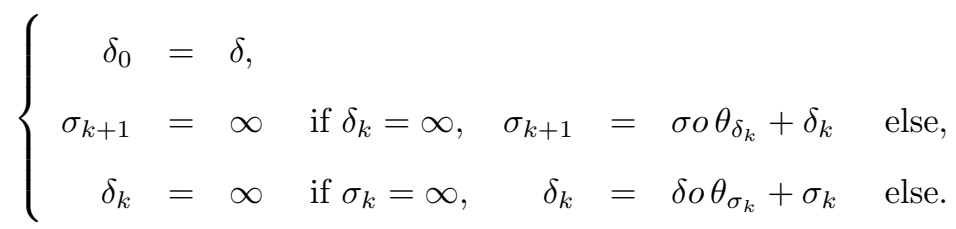

Notice that $\delta_{0}$ is the first time $n$ of having $l_{0}(n)=n$. If $\delta_{k}<\infty$, then $\sigma_{k+1}=\infty$ means that $l_{0}(\infty)=\delta_{k}$. Otherwise, $\sigma_{k+1}<\infty$ is the first time $n>\delta_{k}$ where $l_{0}(n)>\delta_{k}$ and $\delta_{k+1}$ is the first time $n \geq \sigma_{k+1}$ such that $l_{0}(n) \neq \infty$. Actually, $l_{0}\left(\delta_{k+1}\right)=\delta_{k+1}$.

We set

$$
\rho=\sigma+\delta o \theta_{\sigma} .
$$


It follows that

$$
\left.\mathbb{E}\left(e^{\alpha \rho} 1_{\rho<\infty}\right)=\mathbb{E}\left(e^{\alpha \sigma} 1_{\sigma<\infty} \mathbb{E}\left(e^{\alpha \delta} o \theta_{\sigma T} \mid \mathcal{F}_{\sigma T}\right)\right)\right) .
$$

Applying the Markov property and (2.17),

$$
\mathbb{E}\left(e^{\alpha \rho} 1_{\rho<\infty}\right) \leq c \mathbb{E}\left(e^{\alpha \sigma} 1_{\sigma<\infty} \sqrt{1+\mathcal{H}\left(u_{1}(\sigma T)\right)+\mathcal{H}\left(u_{2}(\sigma T)\right)}\right),
$$

and then the Schwarz inequality,

$$
\mathbb{E}\left(e^{\alpha \rho} 1_{\rho<\infty}\right) \leq c \sqrt{\mathbb{E}\left(e^{3 \alpha \sigma} 1_{\sigma<\infty}\right)} \sqrt{\mathbb{E}\left(e^{-\alpha \sigma}\left(1+\mathcal{H}\left(u_{1}(\sigma T)\right)+\mathcal{H}\left(u_{2}(\sigma T)\right)\right) 1_{\sigma<\infty}\right)}
$$

We deduce from $\mathbf{A} 1$ and (2.16) that there exists $C_{0}$ such that

$$
\mathbb{E}\left(e^{\alpha \rho} 1_{\rho<\infty}\right) \leq C_{0},
$$

provided $l_{0}(0)=0$.

Notice that

$$
\delta_{k}=\delta_{k-1}+\rho o \theta_{\delta_{k-1}},
$$

which yields

$$
\mathbb{E}\left(e^{\alpha \delta_{k}} 1_{\delta_{k}<\infty}\right)=\mathbb{E}\left(e^{\alpha \delta_{k-1}} 1_{\delta_{k-1}<\infty} \mathbb{E}\left(e^{\alpha \rho} 1_{\rho<\infty} O \theta_{\delta_{k-1}} \mid \mathcal{F}_{\delta_{k-1} T}\right)\right)
$$

Applying the Markov property, it follows from (2.18) that

$$
\mathbb{E}\left(e^{\alpha \delta_{k}} 1_{\delta_{k}<\infty}\right) \leq C_{0} \mathbb{E}\left(e^{\alpha \delta_{k-1}} 1_{\delta_{k-1}<\infty}\right)
$$

which yields, by (2.17),

$$
\mathbb{E}\left(e^{\alpha \delta_{k}} 1_{\delta_{k}<\infty}\right) \leq c C_{0}^{k}\left(1+\mathcal{H}\left(u_{0}^{1}\right)+\mathcal{H}\left(u_{0}^{2}\right)\right)
$$

We set

$$
k_{0}=\inf \left\{k \in \mathbb{N} \mid \sigma_{k+1}=\infty\right\} .
$$

It follows from (2.15) and from the Markov property that

$$
\mathbb{P}\left(k_{0}>k\right) \leq\left(1-p_{0}\right)^{k} .
$$

Notice that

$$
l_{0}(\infty)=\delta_{k_{0}} .
$$

Let $p \in(1, \infty)$. It follows from Hölder inequality, (2.19) and (2.20) that

$$
\begin{aligned}
\mathbb{E}\left(e^{\frac{\alpha}{p} l_{0}(\infty)}\right) & \leq \sum_{k} \mathbb{E}\left(e^{\frac{\alpha}{p} \delta_{k}} 1_{k_{0}=k}\right) \\
& \leq \sum_{k}\left(\mathbb{E}\left(e^{\alpha \delta_{k}} 1_{\delta_{k}<\infty}\right)\right)^{\frac{1}{p}} \mathbb{P}\left(k_{0}=k\right)^{1-\frac{1}{p}} \\
& \leq c\left(\sum_{k}\left(C_{0}^{\frac{1}{p}}\left(1-p_{0}\right)^{1-\frac{1}{p}}\right)^{k}\right)\left(1+\mathcal{H}\left(u_{0}^{1}\right)+\mathcal{H}\left(u_{0}^{2}\right)\right)
\end{aligned}
$$

Choosing $p$ sufficiently high, we obtain $\gamma^{\prime} \in\left(0, \frac{\gamma}{2 T}\right)$ such that

$$
\mathbb{E}\left(e^{\gamma^{\prime} l_{0}(\infty)}\right) \leq C\left(1+\mathcal{H}\left(u_{0}^{1}\right)+\mathcal{H}\left(u_{0}^{2}\right)\right) .
$$


Exponential Mixing for Stochastic PDEs: The Non-Additive Case.

\subsection{Conclusion.}

Let $t>0$ and $l \in \mathbb{N}$ such that $t \geq l T$. Notice that

$$
\mathbb{E}\left(\left|u_{2}(t)-u_{1}(t)\right| \wedge 1\right) \leq \mathbb{E}\left(\left|u_{2}(t)-u_{1}(t)\right| \wedge 1_{l_{0}(\infty) \leq l}\right)+\mathbb{P}\left(l_{0}(\infty)>l\right) .
$$

Setting $l=\left\lfloor\frac{t}{2 T}\right\rfloor$, it follows from (2.3) and from (2.21)

$$
\mathbb{E}\left(\left|u_{2}(t)-u_{1}(t)\right| \wedge 1\right) \leq C e^{-\gamma^{\prime} t}\left(1+\mathcal{H}\left(u_{0}^{1}\right)+\mathcal{H}\left(u_{0}^{2}\right)\right) .
$$

Now we are able to conclude.

It follows from completeness arguments (See for instance [23]) that, to establish Theorem 2.1] it is sufficient to prove that

$$
\left|\mathcal{P}_{t} f\left(u_{0}^{2}\right)-\mathcal{P}_{t} f\left(u_{0}^{1}\right)\right| \leq C\|f\|_{L} e^{-\gamma^{\prime} t}\left(1+\mathcal{H}\left(u_{0}^{1}\right)+\mathcal{H}\left(u_{0}^{2}\right)\right),
$$

for any $f: H \rightarrow \mathbb{R}$ bounded Lipschitz and any $\left(u_{0}^{1}, u_{0}^{2}, t\right) \in H \times H \times(0, \infty)$.

Since $\left(u_{1}, u_{2}\right)$ is a coupling of $\left(\mathcal{D}\left(u\left(\cdot, W, u_{0}^{1}\right)\right), \mathcal{D}\left(u\left(\cdot, W, u_{0}^{2}\right)\right)\right)$, it follows that $\mathcal{P}_{t} f\left(u_{0}^{i}\right)=\mathbb{E} f\left(u_{i}(t)\right)$ for $i=1,2$ and $t>0$ which yields

$$
\left|\mathcal{P}_{t} f\left(u_{0}^{2}\right)-\mathcal{P}_{t} f\left(u_{0}^{1}\right)\right| \leq 2\|f\|_{L} \mathbb{E}\left(\left|u_{2}(t)-u_{1}(t)\right| \wedge 1\right) .
$$

Then we deduce Theorem 2.1 from (2.22).

\section{The Two-Dimensional Navier-Stokes equations}

In this section, we investigate properties of the two-dimensional Navier-Stokes (NS) equations with Dirichlet boundary conditions. These equations describe the time evolution of an incompressible fluid and are given by

$$
\left\{\begin{array}{rlrl}
d u+\nu(-\Delta) u d t+(u, \nabla) u d t+\nabla p d t & =\phi(u) d W+f d t \\
(\operatorname{div} u)(t, x) & =0, & \text { for } x \in D, t>s, \\
u(t, x) & =0, & \text { for } x \in \partial D, t>s, \\
u(0, x) & =u_{0}(x), & \text { for } x \in D,
\end{array}\right.
$$

where $u(t, x) \in \mathbb{R}^{2}$ denotes the velocity field at time $t$ and position $x, p(t, x)$ denotes the pressure, $\phi(u) d W$ is a random external force field acting on the fluid, $\nu>0$ is the viscosity of the fluid and $D$ is an open bounded domain of $\mathbb{R}^{2}$ with regular boundary or $D=(0,1)^{2}$. Also $f(t, x) d t$ is the deterministic part of the forcing term. For simplicity in the redaction, we consider the case $f=0$. The generalization to a square integrable $f$ is easy.

In Section 3.1 we rewrite problem (3.1) and we state an ergodic result about NS (Theorem 3.4). To establish it, we apply our criterion (Theorem [2.1).

\subsection{Notations and Main result.}

We denote by $|\cdot|$ the norm of $L^{2}\left(D ; \mathbb{R}^{2}\right)$ and by $\|\cdot\|$ the norm of $H_{0}^{1}\left(D ; \mathbb{R}^{2}\right)$. Let $H$ and $V$ be the closure of the space of smooth functions on $D$ with compact support and free divergence for the norm $|\cdot|$ and $\|\cdot\|$, respectively.

Let $\Pi$ be the orthogonal projection in $L^{2}\left(D ; \mathbb{R}^{2}\right)$ onto the space $H$. Setting

$$
A=\Pi(-\Delta), \quad D(A)=V \cap H^{2}\left(D ; \mathbb{R}^{2}\right) \text { and } B(u)=\Pi((u, \nabla) u),
$$

we can write problem (3.1) in the form

$$
\left\{\begin{aligned}
d u+\nu A u d t+B(u) d t & =\phi(u) d W \\
u(0) & =u_{0},
\end{aligned}\right.
$$

where $W$ is a cylindrical Wiener process on a Hilbert space $U$. 
In order to have existence and uniqueness of the solution of (3.2), we make the following assumption

H0 The function $\phi: H \rightarrow \mathcal{L}_{2}(U ; H)$ is bounded Lipschitz.

We set

$$
B_{0}=\sup _{u \in H}\|\phi(u)\|_{\mathcal{L}_{2}(U ; H)}^{2}, \quad L=L_{\phi}^{2},
$$

where $L_{\phi}$ is the Lipschitz constant of $\phi$.

There exists a unique $H$-valued solution $u\left(\cdot, W, u_{0}\right)$ of (3.2). This is a continuous homogenous weak Markov process and a non anticipative measurable map of $W$. Moreover the law $\mathcal{D}\left(u\left(\cdot, W, u_{0}\right)\right)$ measurably depends of $u_{0}$. Existence can be established by using Galerkin approximation. To prove uniqueness and measurability with respect to $u_{0}$, it is possible to prove the last inequality of Remark 1.3.

Remark 3.1. Using Jenji-Krylov arguments, it is possible to prove that there exists a measurable mapping $\left(W, u_{0}\right) \rightarrow u\left(\cdot, W, u_{0}\right)$ with value in continuous mapping $(0, \infty) \rightarrow H$ such that $u\left(\cdot, W, u_{0}\right)$ is the unique solution of (3.2). However, we do not need it here.

We denote by $\left(\mathcal{P}_{t}\right)_{t \in \mathbb{R}^{+}}$the Markov transition semi-group associated to the Markov family $\left(u\left(\cdot, W, u_{0}\right)\right)_{u_{0} \in H}$.

In our computations, we use the following energy

$$
E_{u}(t)=|u(t)|^{2}+\nu \int_{0}^{t}\|u(s)\|^{2} d s .
$$

It is well-known that $(A, \mathcal{D}(A))$ is a self-adjoint operator with discrete spectrum. See 4], 32. We consider $\left(e_{n}\right)_{n}$ an eigenbasis of $H$ associated to the increasing sequence $\left(\mu_{n}\right)_{n}$ of eigenvalues of $(A, \mathcal{D}(A))$. We denote by $P_{N}$ and $Q_{N}$ the orthogonal projection in $H$ onto the space $S p\left(e_{k}\right)_{1 \leq N}$ and onto its complementary, respectively.

Now, we make the assumption which is used to prove the exponential mixing of $\left(\mathcal{P}_{t}\right)_{t \in \mathbb{R}^{+}}$.

H1 There exist $N \in \mathbb{N}^{*}$ and a bounded measurable map $g: H \rightarrow \mathcal{L}(H ; U)$ such that for any $u \in H$

$$
\phi(u) g(u)=P_{N} .
$$

Remark 3.2 (Sufficient conditions to satisfy H1).

A sufficient condition to satisfy $\mathbf{H 1}$ is for instance that $U$ is the orthogonal sum $U_{1} \oplus U_{2}$ and there exist two measurable maps $\left(\phi_{i}: H \rightarrow \mathcal{L}_{2}\left(U_{i} ; H\right)\right)_{i=1,2}$ such that $\phi_{2}$ verifies $\mathbf{H} \mathbf{1}$ and

$$
\phi(u) W=\phi_{1}(u) W_{1}+\phi_{2}(u) W_{2},
$$

for any $u \in H$ and any $W=\left(W_{1}, W_{2}\right) \in U$. Moreover, if $\phi_{2}$ is a constant map, we can omit the orthogonality condition on $U_{1}$ and $U_{2}$.

A very interesting consequence is the case of the sum of a multiplicative noise and an additive noise which covers the low modes. Namely, for any measurable map $f: \mathbb{R}^{2} \rightarrow \mathbb{R}$ and $\left(\phi_{1}, \phi_{2}\right) \in \mathcal{L}_{2}\left(U_{1} ; H^{2}(D)\right) \times \mathcal{L}_{2}\left(U_{2} ; H\right)$, one define $\phi$ by

$$
\phi(u) W=f(u)\left(\phi_{1} W_{1}\right)+\phi_{2} W_{2},
$$


Exponential Mixing for Stochastic PDEs: The Non-Additive Case.

for any $u \in H$ and any $W=\left(W_{1}, W_{2}\right) \in U$. The operator $\phi$ verifies $\mathbf{H 1}$ provided

$$
P_{N} H \subset \operatorname{Im} \phi_{2} \text {. }
$$

Another sufficient condition is that $U=H$ and there exists an invertible operator $\psi$ on the low modes and a constant $\varepsilon$ such that for any $u \in H$

$$
Q_{N} \phi(u) P_{N}=0 \quad \text { and } \quad\left|P_{N} \phi(u) P_{N}-\psi\right|_{\mathcal{L}\left(P_{N} H\right)}<\varepsilon<\left|\psi^{-1}\right|_{\mathcal{L}\left(P_{N} H\right)}^{-1} .
$$

Thus, our result holds when the covariance operator is a small perturbation of a constant in the low modes.

We think that these examples might be physically relevant.

Remark 3.3. The existence of a map $\widetilde{g}(u)$ such that $\phi(u) \widetilde{g}(u)=P_{N}$, is equivalent to the following property

$$
P_{N} H \subset \operatorname{Im}(\phi(u)) .
$$

Hence $\mathbf{H 1}$ can be seen as a non degeneracy condition on the low modes in the spirit of 3, 8, 9], 14, 19, 21, 22, 23, 26, 30] and 31. The lack of surjectivity of $\phi(u)$ on the high modes is counterbalanced by the dissipativity of (3.2).

Moreover, if there exists such a map $\widetilde{g}$, then there exists a measurable map $g$ such that $\phi(u) g(u)=P_{N}$ and $|g(u)|_{\mathcal{L}(H ; U)} \leq|\widetilde{g}(u)|_{\mathcal{L}(H ; U)}$. This mapping $g$ is constructed by similar ideas as in the construction of the pseudo inverse. Hence the assumption of measurability of $g$ in $\mathbf{H 1}$ is superfluous.

The aim of this section is to prove the following result.

Theorem 3.4. Assume that $\mathbf{H O}$ holds. There exists $N_{0}\left(B_{0}, \nu, D, L\right)$ such that, if $\mathbf{H 1}$ holds with $N \geq N_{0}$, then there exists a unique stationary probability measure $\mu$ of $\left(\mathcal{P}_{t}\right)_{t \in \mathbb{R}^{+}}$on $H$. Moreover, $\mu$ satisfies

$$
\int_{H}|u|^{2} d \mu(u)<\infty
$$

and there exist $C, \gamma>0$, such that for any $\lambda \in \mathcal{P}(H)$

$$
\left\|\mathcal{P}_{t}^{*} \lambda-\mu\right\|_{*} \leq C e^{-\gamma t}\left(1+\int_{H}|u|^{2} d \lambda(u)\right) .
$$

The remaining subsections of this section are devoted to prove Theorem [3.4 by applying Theorem 2.1.

The proof of our result is based on coupling arguments. These arguments have initially been used in the context of dissipative SPDEs in [23, [26]. For a better understanding of this kind of method, see section 1 of [26] and the two first subsections of [30]. There the coupling method is explained on two examples which are simpler but contain all the difficulties.

The method used in 23] and [26] requires the three following assumptions for a $N$ sufficiently high.

The first assumption is a structure condition on $\phi$. It is a slight generalization of the usual assumption that $\phi(u)$ is diagonal in the basis $\left(e_{n}\right)_{n}$.

Ha The Hilbert space $U$ is $H$. Moreover, for any $u \in H$, we have

$$
P_{N} \phi(u) Q_{N}=0, Q_{N} \phi(u) P_{N}=0 .
$$

The second assumption means that $\phi$ only depends on its low modes.

Hb For any $u \in H$

$$
\phi(u)=\phi\left(P_{N} u\right)
$$


Exponential Mixing for Stochastic PDEs: The Non-Additive Case.

The third assumption is $\mathbf{H 1}$. Under $\mathbf{H a}$, it could be written in the form.

Hc The linear map $P_{N} \phi(u) P_{N}$ is invertible on $P_{N} H$. Moreover

$$
\sup _{u \in H}\left|\left(P_{N} \phi(u) P_{N}\right)^{-1}\right|_{\mathcal{L}\left(P_{N} H ; P_{N} H\right)}<\infty .
$$

In these papers, the proof is divided in two steps.

Step 1: Starting from initial data $\left(u_{0}^{1}, u_{0}^{2}\right)$ in a ball of radius $R_{0}$, Girsanov transform can be used to show that there exists a couple of Wiener processes $\left(W_{1}, W_{2}\right)$ such that

$$
P_{N} u\left(T, W_{1}, u_{0}^{1}\right)=P_{N} u\left(T, W_{2}, u_{0}^{2}\right)
$$

with positive probability.

Step 2: Another ingredient, the so-called Foias-Prodi estimate, is used. It is based on the observation that if two solutions are such that

$$
\left(P_{N} u\left(\cdot, W_{1}, u_{0}^{1}\right), Q_{N} W_{1}\right)=\left(P_{N} u\left(\cdot, W_{2}, u_{0}^{2}\right), Q_{N} W_{2}\right),
$$

for a long time then they become very close. Girsanov transform can again be used to show that, if we start from initial conditions which have the same low modes, then there exists a couple of Wiener processes $\left(W_{1}, W_{2}\right)$ such that the low modes of the solutions remain equal for all times with a positive probability.

Conclusion: Since the time of entering a ball of radius $R_{0}$ admits an exponential moment, we are able to combine and to iterate the two steps and then to conclude.

In the above mentioned articles, $\mathbf{H a}$ and $\mathbf{H b}$ are essential when using the Girsanov transform. They are also necessary to prove that the Foais-Prodi estimate holds pathwise. This latter point is not important. We have shown in [8 how to use a Foias-Prodi estimate in expectation and it is not difficult to see that such an estimate holds in our case.

On the contrary, it seems very difficult to use Girsanov Transform without Ha and $\mathbf{H b}$. We think that these are artificial whereas $\mathbf{H} \mathbf{1}$ is very natural.

The idea used in this article is to separate the use of the Foias-Prodi estimate and the Girsanov Transform by introducing an auxiliary process $\widetilde{u}\left(\cdot, W, u_{0}^{1}, u_{0}^{2}\right)$ (In some some sense, this idea could be related to the binding processes of [14]). Our criterion (Theorem 2.1) essentially requires that the auxiliary process verifies two properties.

First fundamental property: The first property is a variation of the Foias-Prodi estimate. It states that $u\left(t, W, u_{0}^{1}\right)$ and $\widetilde{u}\left(t, W, u_{0}^{1}, u_{0}^{2}\right)$ become close exponentially fast in probability.

Second fundamental property: The second property is $\mathbf{A 3}$ and states that there exists $h$ such that

$$
u\left(t, W, u_{0}^{1}, u_{0}^{2}\right)=u\left(t, W+\int_{0} h\left(u\left(s, W, u_{0}^{1}\right), \widetilde{u}\left(s, W, u_{0}^{1}, u_{0}^{2}\right)\right) d s, u_{0}^{2}\right) .
$$

Hence, taking into account H1, it follows from coupling arguments and from a Girsanov Transform that there exists a couple of Wiener processes $\left(W_{1}, W_{2}\right)$ such that

$$
\widetilde{u}\left(\cdot, W_{1}, u_{0}^{1}, u_{0}^{2}\right)=u\left(\cdot, W_{2}, u_{0}^{2}\right),
$$

with a positive probability.

Conclusion: Iterating and combining the two properties, we can conclude by remarking that it allows to control the probability of $u\left(t, W_{1}, u_{0}^{1}\right)$ and $u\left(t, W_{2}, u_{0}^{2}\right)$ being very close. 


\subsection{Energy estimate and Lyapunov structure.}

Setting $\mathcal{H}=|\cdot|^{2}$, the following result states that $\mathbf{A} \mathbf{1}$ is true.

Lemma 3.5 (The Lyapunov structure). Assume Ho. There exist $C_{1}$ and a family $\left(C_{\alpha}^{\prime}\right)_{\alpha}$ only depending on $\nu, B_{0}$ and $D$ such that

$$
\left\{\begin{aligned}
\mathbb{E}\left(\left|u\left(t, W, u_{0}\right)\right|^{2}\right) & \leq e^{-\nu \mu_{1} t}\left|u_{0}\right|^{2 q}+C_{1}, \\
\mathbb{E}\left(e^{-\alpha \tau}\left|u\left(\tau, W, u_{0}\right)\right|^{2} 1_{\tau<\infty}\right) & \leq\left|u_{0}\right|^{2}+C_{\alpha}^{\prime},
\end{aligned}\right.
$$

for any $t \geq 0$, any $\alpha>0$ and any stopping time $\tau$.

Proof of Lemma 3.5

We set $u=u\left(\cdot, W, u_{0}\right)$. We apply Ito Formula to $|u|^{2}$

$$
d|u|^{2}+2 \nu\|u\|^{2} d t+2(u, B(u)) d t=2(u, \phi(u) d W)+\|\phi(u)\|_{\mathcal{L}_{2}(U ; H)}^{2} d t .
$$

Recall that

$$
(u, B(u))=0 .
$$

Hence, taking into account Ho, we deduce

$$
d|u|^{2}+2 \nu\|u\|^{2} d t=2(u, \phi(u) d W)+B_{0} d t .
$$

Notice that $\|\cdot\|^{2} \geq \mu_{1}|\cdot|^{2}$. Hence, integrating and taking the expectation, we establish the first inequality of Lemma 3.5.

Let $\alpha>0$. Ito Formula of $e^{-\alpha t}|u(t)|^{2}$ gives

$$
d e^{-\alpha s}|u|^{2}+e^{-\alpha s}\left(2 \nu\|u\|^{2}+\alpha|u|^{2}\right) d t=2 e^{-\alpha s}(u, \phi(u) d W)+B_{0} e^{-\alpha s} d t .
$$

Let $\tau$ be a stopping time and $n \in \mathbb{N}$. Taking the expectation, it follows

$$
\mathbb{E}\left(e^{-\alpha \tau \wedge n}|u(\tau \wedge n)|^{2}\right) \leq\left|u_{0}\right|^{2}+C_{\alpha}^{\prime} .
$$

Let $n \rightarrow \infty$, the second inequality of Lemma 3.5

The next result will be useful in the following.

Lemma 3.6 (Exponential estimate for the growth of the solution). Assume that H0 holds. There exists $\gamma_{0}>0$ only depending on $\nu, B_{0}$ and $D$ such that

$$
\mathbb{E}\left(\exp \left(\gamma_{0} \sup _{t \geq 0}\left(E_{u\left(\cdot, W, u_{0}\right)}(t)-B_{0} t\right)\right)\right) \leq 2 e^{\gamma_{0}\left|u_{0}\right|^{2}},
$$

for any $u_{0} \in H$.

Proof of Lemma 3.6

We set for any $\gamma>0$

$$
M(t)=2 \int_{0}^{t}(u(r), \phi(u(r)) d W(r)), \quad \mathcal{M}_{\gamma}(t)=M(t)-\frac{\gamma}{2}\langle M\rangle(t) .
$$

Remarking that

$$
d\langle M\rangle=4\left|\phi(u)^{*} u\right|^{2} d t \leq 4 c B_{0}\|u\|^{2} d t,
$$

and setting $\gamma_{1}=\frac{\nu}{2 c B_{0}}$, we deduce from (3.5) that

$$
E_{u}(t) \leq \mathcal{M}_{\gamma_{1}}(t)+\left|u_{0}\right|^{2}+B_{0} t
$$


Notice that $e^{\gamma \mathcal{M}_{\gamma}}$ is a positive supermartingale whose value is 1 at time 0 . We deduce from maximal supermartingale inequality that

$$
\mathbb{P}\left(\sup _{t \geq 0} \mathcal{M}_{\gamma_{1}}(t) \geq \rho\right) \leq \mathbb{P}\left(\sup _{t \geq 0} e^{\gamma_{1} \mathcal{M}_{\gamma_{1}}(t)} \geq e^{\gamma_{1} \rho}\right) \leq e^{-\gamma_{1} \rho} .
$$

We set $\gamma_{0}=\frac{\gamma_{1}}{2}$. Notice that

$$
\mathbb{E}\left(e^{\gamma_{0} \sup \mathcal{M}_{\gamma_{1}}}\right)=1+\gamma_{0} \int_{0}^{\infty} e^{\gamma_{0} x} \mathbb{P}\left(\sup \mathcal{M}_{\gamma_{1}} \geq x\right) d x,
$$

which yields, by (3.7),

$$
\mathbb{E}\left(e^{\gamma_{0} \sup \mathcal{M}_{\gamma_{1}}}\right) \leq 2
$$

Combining (3.6) and (3.8), it follows

$$
\mathbb{E}\left(\exp \left(\gamma_{0} \sup _{t \geq 0}\left(E_{u\left(\cdot, W, u_{0}\right)}(t)-B_{0} t\right)\right)\right) \leq 2 e^{\gamma_{0}\left|u_{0}\right|^{2}} .
$$

This ends the proof of Lemma 3.6

\subsection{Construction of the auxiliary process.}

Now, we build the auxiliary process. We set

$$
F(u)=\nu A(u)+B(u) .
$$

Taking into account $\mathbf{H 1}$, we remark that $\mathbf{A} \mathbf{3}$ is a consequence of

$$
\left\{\begin{aligned}
d \widetilde{u}+F(\widetilde{u}) d t+P_{N} \delta\left(u\left(t, W, u_{0}\right), \widetilde{u}\right) d t & =\phi(\widetilde{u}) d W, \\
\widetilde{u}\left(0, W, u_{0}, \widetilde{u}_{0}\right) & =\widetilde{u}_{0},
\end{aligned}\right.
$$

with

$$
h(u, \widetilde{u})=-g(\widetilde{u}) \delta(u, \widetilde{u}) .
$$

Since we want that $\widetilde{u}\left(t, W, u_{0}^{1}, u_{0}^{2}\right)$ and $u\left(t, W, u_{0}^{1}\right)$ become very close in probability, it is natural to build $\widetilde{u}$ such that (3.9) and (3.10) hold with

$$
\delta(u, \widetilde{u})=K P_{N}(\widetilde{u}-u) .
$$

Hence we consider the following equation

$$
\left\{\begin{aligned}
d \widetilde{u}+F(\widetilde{u}) d t+K P_{N}\left(\widetilde{u}-u\left(t, W, u_{0}^{1}\right)\right) d t & =\phi(\widetilde{u}) d W, \\
\widetilde{u}(0) & =\widetilde{u}_{0},
\end{aligned}\right.
$$

where $K>0$ will be chosen later and $N$ is the integer used in H1.

As for (3.2), we deduce from $\mathbf{H 0}$ that there exists a unique $H$-valued solution to (3.12) when $\left(u_{0}, \widetilde{u}_{0}\right) \in H^{2}$. Moreover, $\widetilde{u}$ is a non anticipative measurable map of $W$ (See Remark 1.2). It follows from the uniqueness that $(u, \widetilde{u})$ is a homogenous weak Markov process. Moreover, its law $\mathcal{D}(u, \widetilde{u})$ is measurably depending of its initial condition $\left(u_{0}, \widetilde{u}_{0}\right)$ (To prove it, one can establish the last inequality of Remark 1.3. Arguments are similar but simpler than those of the proof of the next proposition).

We will denote the dependance of $(u, \widetilde{u})$ with respect to $\left(t, W, u_{0}, \widetilde{u}_{0}\right)$ as follows

$$
(u(t), \widetilde{u}(t))=\left(u\left(t, W, u_{0}\right), \widetilde{u}\left(t, W, u_{0}, \widetilde{u}_{0}\right)\right)
$$

and we deduce A0.

Taking into account $\mathbf{H 1}$ and the uniqueness of the solution of (3.2) under Ho, we deduce A3 with (3.10) and (3.11). 
Exponential Mixing for Stochastic PDEs: The Non-Additive Case.

Now, it remains to prove $\mathbf{A 2}, \mathbf{A} 4$ and $\mathbf{A 5}$, i.e. that $\widetilde{u}\left(t, W, u_{0}, \widetilde{u}_{0}\right)$ and $u\left(t, W, u_{0}\right)$ become close exponentially fast. In [23, 26] and 30, a pathwise Foias-Prodi is used. Here, $u-\widetilde{u}$ does not seem to tend to 0 , pathwise. That is the reason why we adapt an idea we have already used in 8 to prove polynomial mixing for the weakly damped Non-linear Schrödinger (NLS) equations. Since it seems that there is no pathwise Foias-Prodi estimate for NLS, a Foias-Prodi estimate in expectation was used. Using analogous technics, we have the following result.

Proposition 3.7. Assume that $\mathbf{H 0}$ holds. There exist $\gamma>0, \varepsilon \in(0,1], K_{0}>0$ and $N_{0}$ depending only on $B_{0}, \nu$ and $D$ such that for any $K \geq K_{0}$ and $N \geq N_{0}$ and any $\left(t, u_{0}, \widetilde{u}_{0}\right) \in(0, \infty) \times H^{2}$

$$
\mathbb{E}\left(\left(e^{t}|r(t)|^{2}+\int_{0}^{t} e^{s}\|r(s)\|^{2} d s\right)^{\varepsilon}\right) \leq 2|r(0)|^{2 \varepsilon} e^{\gamma\left|u_{0}\right|^{2}},
$$

where

$$
r=\widetilde{u}\left(\cdot, W, u_{0}, \widetilde{u}_{0}\right)-u\left(\cdot, W, u_{0}\right) .
$$

Notice that, by Chebyshev inequality, this result obviously implies A2, A4 and A5.

Proof of Proposition 3.7

For any function $f$, we denote by $\delta f(u)$ the value $f(\widetilde{u})-f\left(u_{1}\right)$. Taking the difference between (3.2) and (3.12), we obtain

$$
d r+\nu A r d t+K P_{N} r d t+\delta B(u) d t=\delta \phi(u) d W .
$$

Hence, applying Ito Formula to $|r|^{2}$, we have

$$
d|r|^{2}+2\left(\nu\|r\|^{2}+K\left|P_{N} r\right|^{2}\right) d t=2(r, \delta \phi(u) d W)+I(t) d t
$$

where

$$
I(t)=I_{1}(t)+I_{2}(t), \quad I_{1}(t)=-2(r, \delta B(u)), \quad I_{2}(t)=\|\delta \phi(u)\|_{\mathcal{L}_{2}(U ; H)}^{2} .
$$

Remarking that

$$
\delta B(u)=\pi((\widetilde{u}, \nabla) r+(r, \nabla) u), \quad(r,(\widetilde{u}, \nabla) r)=0,
$$

we deduce from a Schwartz inequality that

$$
I_{1}(t) \leq c\left|r^{2}\right|\|u\|=c|r|_{4}^{2}\|u\| .
$$

It follows from Sobolev Embedding $H^{\frac{1}{2}}\left(D, \mathbb{R}^{2}\right) \subset L^{4}\left(D, \mathbb{R}^{2}\right)$ and interpolatory inequality that

$$
I_{1}(t) \leq c\|r\|_{\frac{1}{2}}^{2}\left\|u_{1}\right\| \leq c\|r\||r|\|u\| .
$$

We infer from an arithmetico-geometric inequality that

$$
I_{1}(t) \leq \nu\|r\|^{2}+c|r|^{2}\|u\|^{2} .
$$

Applying Ho, we obtain

$$
I_{2}(t) \leq L|r|^{2}
$$

Remarking that

$$
\left(K \wedge\left(\nu \mu_{N+1}\right)\right)|r|^{2} \leq \nu\left\|Q_{N} r\right\|^{2}+K\left|P_{N} r\right|^{2} \leq \nu\|r\|^{2}+K\left|P_{N} r\right|^{2},
$$

we deduce from (3.13), (3.14) and (3.15) that there exists $\Lambda$ such that

$$
d|r|^{2}+\left(\left(K \wedge\left(\nu \mu_{N+1}\right)-L\right)-\Lambda\left\|u_{1}\right\|^{2}\right)|r|^{2} d t \leq 2(r, \delta \phi(u) d W) .
$$


Integrating this formula and taking the expectation, it follows

$$
\mathbb{E}\left(e^{t} G(t)^{-1}|r(t)|^{2}+\int_{0}^{t} e^{s} G(s)^{-1}\|r(s)\|^{2} d s\right) \leq|r(0)|^{2},
$$

where

$$
G(t)=e^{-\left(K \wedge\left(\nu \mu_{N+1}\right)-L-1\right) t+\Lambda \int_{0}^{t}\|u(r)\|^{2} d r} .
$$

It follows from Hölder inequality that

$$
\begin{aligned}
& \mathbb{E}\left(\left(e^{t}|r(t)|^{2}+\int_{0}^{t} e^{s}\|u(s)\|^{2} d s\right)^{\varepsilon}\right) \leq \sqrt{\mathbb{E}\left(\sup G^{2 \varepsilon}\right)} \times \\
& \left(\mathbb{E}\left(e^{t} G(t)^{-1}|r(t)|^{2}+\int_{0}^{t} e^{s} G(s)^{-1}\|r(s)\|^{2} d s\right)\right)^{\varepsilon} .
\end{aligned}
$$

Choosing $N, K$ sufficiently high and $\varepsilon>0$ sufficiently small, it follows from Lemma 3.6 that

$$
\mathbb{E}\left(\sup G^{2 \varepsilon}\right) \leq 2 e^{\gamma_{0}\left|u_{0}\right|^{2}}
$$

which yields Proposition 3.7

\subsection{Conclusion and Remarks.}

We have proved that assumptions of Theorem 2.1 are verified. So Theorem 3.4 follows.

Actually, A0 is a consequence of the well-posedness of equations.

We set $\mathcal{H}=|\cdot|^{2}$. As shown in Lemma 3.5] we have $\mathbf{A 1}$.

Assumption A3 has been proved at the beginning of section 3.3.

We deduce A2, A4 and A5 directly from Proposition 3.7

Since A0,.., A5 are established, we can apply Theorem 2.1 which yields Theorem 3.4

Remark 3.8. Theorem 3.4 could be improved and the assumptions could be weakened. We chose to restrict to this statement for clarity and readability. For instance, it is possible to replace $\mathbf{H} \mathbf{1}$ by

H1' There exist $n \in \mathbb{N}^{*}$, a measurable map $g: H \rightarrow \mathcal{L}(H ; U)$ and two constants $\sigma, C$ such that for any $u \in H$

$$
\phi(u) g(u)=P_{N}, \quad|g(u)|_{\mathcal{L}(H ; U)} \leq C \exp \left(\sigma|u|^{2}\right) .
$$

In this case $N_{0}$ depends on $\sigma$. Moreover, it is easy to strengthen (3.3) into

$$
\int_{H} \exp \left(\sigma_{1}\left(B_{0}, \nu, D\right)|u|^{2}\right) d \mu(u)<\infty .
$$

In HO, the boundedness of $\phi$ could be replaced by $|\phi(u)| \leq C\left(1+|u|^{\gamma}\right)$ with $\gamma<1$. In this case, the rate of convergence becomes greater than any power of time instead of being exponential in time. Moreover for any $p$ there exists $c_{p}$ such that if there exists $C$ such that $|\phi(u)| \leq C+c_{p}|u|$, then the rate of convergence is greater than $(1+t)^{-p}$ instead of being exponential.

Assume now that $D$ is the two-dimensional torus. We replace the Dirichlet boundary condition by periodic condition and we assume that $\mathbf{H 0}$ and $\mathbf{H 1}$ ' hold for $N$ sufficiently high and

$$
B_{1}=\sup _{u \in H}\|\phi(u)\|_{\mathcal{L}_{2}(U ; V)}^{2}<\infty
$$


Exponential Mixing for Stochastic PDEs: The Non-Additive Case.

then we can strengthen (3.3) by

$$
\int_{H} \exp \left(\sigma_{2}\left(B_{1}, \nu, D\right)\|u\|^{2}\right) d \mu(u)<\infty
$$

and in (3.4) we can replace $\|\cdot\|_{*}$, the Wassertein norm in $H$, by the Wassertstein norm in $H^{s}\left(D ; \mathbb{R}^{2}\right)$ for any $s<1$. Moreover, if $\phi: H \rightarrow \mathcal{L}_{2}(U ; V)$ is bounded Lipschitz, then in (3.4) we can replace $\|\cdot\|_{*}$ the Wassertein norm in $H$ by the Wassertstein norm in $V$.

Remark 3.9. Theorem 3.4 is of the same type as the results obtained in [9], 23], 26] and 30] where SPDEs with additive noise only depending on the low modes are studied. Since the decrease of $\mathcal{P}_{t}^{*} \lambda-\mu$ is measured in Wasserstein norm, we know that $\mathcal{P}_{t} \phi$ converges to its average with respect to $\mu$ for any Lipschitz function $\phi$. In fact, in the above mentioned articles, it is also true if $\phi$ is only Lipschitz with respect to the high modes. We do not know if this holds in our situation.

Remark 3.10. Proof of Proposition 3.7 displays a nice and well known property of Navier-Stokes equations. Indeed, we see that the difference between $u$ and $\widetilde{u}$ can be estimated using only the energy of $u$ and not the energy of $\widetilde{u}$ which is much more difficult to estimate. No control on the probability of the linear growth of the energy of the auxiliary process is required. This property holds also for the one-dimensional Burgers equation and for the Complex Ginzburg-Landau equation with a globally Lipschitz noise in the subcritical case. However it does not hold in general and in this case the construction of the auxiliary process is more involved. For instance, in the case of the Non-Linear Schrödinger equations, it is not possible to prove a result similar to Proposition 3.7. It is important to show that our method can also be used for these equations. The Non-Linear Schrödinger equations will be treated in a forthcoming article and we consider the Complex Ginzburg-Landau with a locally Lipschitz noise in section 4.

\section{The Complex Ginzburg-Landau equation with a locally Lipschitz NOISE}

The aim of this section is to apply our method to the stochastic CGL equation with Dirichlet boundary conditions and with a locally Lipschitz noise.

Let us recall that it has the form

$$
\left\{\begin{aligned}
d u+(\varepsilon+\mathrm{i})(-\Delta) u d t+(\eta+\lambda \mathrm{i})|u|^{2 \sigma} u d t & =\phi(u) d W+f d t, \\
u(t, x) & =0, \text { for } x \in \partial D, t>0, \\
u(0, x) & =u_{0}(x), \text { for } x \in D,
\end{aligned}\right.
$$

where $D$ is an open bounded domain of $\mathbb{R}^{d}$ with regular boundary or $D=(0,1)^{d}$, where $\varepsilon>0, \eta>0, \lambda \in\{-1,1\}$ and where we impose the $L^{2}$-subcritical condition $\sigma d<2$. For simplicity in the redaction, we consider the case $f=0$, where $f$ is the deterministic part of the forcing term $\phi(u) d W+f d t$. The generalization to a square integrable $f$ is easy.

Ergodicity for the stochastic CGL equation is established in 1] when the noise is invertible and in 14 for the one-dimensional cubic case when the noise is diagonal, does not depend on the solution and is smooth in space. Then, in 30, we have established exponential mixing of CGL driven by a noise which verifies $\mathbf{H a}, \mathbf{H b}$ and $\mathbf{H c}$ under the $L^{2}$ or the $H^{1}$-subcritical conditions. 
Exponential Mixing for Stochastic PDEs: The Non-Additive Case.

As explained in Remark 3.10] technics of section 3 can easily be applied to the stochastic CGL equation with a globally Lipschitz noise. It gives exponential mixing in $L^{2}$ under the $L^{2}$-subcritical condition $\sigma d<2$. Moreover, one can obtain the exponential mixing in $H^{1}$ under the $H^{1}$-subcritical condition $(d-2) \sigma<2$ when $\lambda=1$. Using a polynomial version of our criterion, one can obtain polynomial mixing in $H^{1}$ under the $L^{2}$-subcritical condition $\sigma d<2$ when $\lambda=-1$.

As explained in Remark 3.10, it seems that such technics can not always be applied when there is no analogous property to Proposition 3.7. For instance, the case of the stochastic non-linear Schrödinger equation requires more sophisticated tools and will be treated in a forthcoming paper. We study the CGL equation with a locally Lipschitz noise because it gives a simple example of SPDE for which the difference of two solutions cannot be estimated with the help of only one energy, an essential ingredient in the proof of Proposition 3.7

\subsection{Notations and Main result.}

We set

$$
H=L^{2}(D ; \mathbb{C}), \quad A=-\Delta, \quad D(A)=H_{0}^{1}(D ; \mathbb{C}) \cap H^{2}(D ; \mathbb{C}),
$$

and we denote by $|\cdot|,|\cdot|_{p},\|\cdot\|$ and $\|\cdot\|_{s}$ the norm of $\mathbb{C}, L^{p}(D ; \mathbb{C}), H_{0}^{1}(D ; \mathbb{C})$ and $H^{s}(D ; \mathbb{C})$. The norm of $H$ will be denoted by $|\cdot|$ when no confusion is possible or $|\cdot|_{H}$ otherwise.

Now we can write problem (4.1) in the form

$$
\left\{\begin{aligned}
d u+(\varepsilon+\mathrm{i}) A u d t+(\eta+\lambda \mathrm{i})|u|^{2 \sigma} u d t & =\phi(u) d W \\
u(0) & =u_{0},
\end{aligned}\right.
$$

where $W$ is a cylindrical Wiener process on a Hilbert $U$.

In order to have existence and uniqueness of the solution of (4.2), we make the following assumption

H0' The function $\phi: H \rightarrow \mathcal{L}_{2}(U ; H)$ is bounded and local Lypschitz. More precisely, we assume there exists $L>0$ such that for any $\left(u_{1}, u_{2}\right) \in H^{2}$

$$
\left\|\phi\left(u_{2}\right)-\phi\left(u_{1}\right)\right\|_{\mathcal{L}_{2}(U ; H)}^{2} \leq L\left|u_{2}-u_{1}\right|^{2}\left(1+\left|u_{1}\right|^{2 \sigma}+\left|u_{2}\right|^{2 \sigma}\right) .
$$

We set

$$
B_{1}=\sup _{u \in H}\|\phi(u)\|_{\mathcal{L}_{2}(U ; H)}^{2} .
$$

Under H0', there exists a unique $H$-valued solution $u\left(\cdot, W, u_{0}\right)$ of (4.2). This is a continuous homogenous weak Markov process and a non anticipative measurable map of $W$. Moreover the law $\mathcal{D}\left(u\left(\cdot, W, u_{0}\right)\right)$ measurably depends of $u_{0}$. Existence, uniqueness and measurable dependance can be established by a contracting fixpoint argument applied to the mild form of (4.2).

We denote by $\left(\mathcal{P}_{t}\right)_{t \in \mathbb{R}^{+}}$the Markov transition semi-group associated to the Markov family $\left(u\left(\cdot, W, u_{0}\right)\right)_{u_{0} \in H}$.

In our computations, we use the following energy

$$
E_{u}(t)=|u(t)|^{2}+\varepsilon \int_{0}^{t}\|u(s)\|^{2} d s+\eta \int_{0}^{t}|u(s)|_{2 \sigma+2}^{2 \sigma+2} d s .
$$

It is well-known that $(A, \mathcal{D}(A))$ is a self-adjoint operator with discrete spectrum. We consider $\left(e_{n}\right)_{n}$ an eigenbasis of $H$ associated to the increasing sequence $\left(\mu_{n}\right)_{n}$ of eigenvalues of $(A, \mathcal{D}(A))$. We denote by $P_{N}$ and $Q_{N}$ the orthogonal projection in $H$ onto the space $S p\left(e_{k}\right)_{1 \leq N}$ and onto its complementary, respectively. 
Now, we state the assumption which gives the exponential mixing of $\left(\mathcal{P}_{t}\right)_{t \in \mathbb{R}^{+}}$provided it holds for $N$ sufficiently high.

H1 There exists a bounded measurable map $g: H \rightarrow \mathcal{L}(H ; U)$ such that for any $u \in H$

$$
\phi(u) g(u)=P_{N} .
$$

The aim of this section is to establish the following result.

Theorem 4.1. Assume H0'. There exists $N_{0}\left(B_{1}, \varepsilon, \eta, \sigma, D, L\right)$ such that, if $\mathbf{H 1}$ holds with $N \geq N_{0}$, then there exists a unique stationary probability measure $\mu$ of $\left(\mathcal{P}_{t}\right)_{t \in \mathbb{R}^{+}}$on $H$. Moreover, $\mu$ satisfies

$$
\int_{H}|u|^{2} d \mu(u)<\infty
$$

and there exist $C, \gamma>0$ such that for any $\lambda \in \mathcal{P}(H)$

$$
\left\|\mathcal{P}_{t}^{*} \lambda-\mu\right\|_{*} \leq C e^{-\gamma t}\left(1+\int_{H}|u|^{2} d \lambda(u)\right) .
$$

We now prove prove Theorem 4.1 by applying Theorem 2.1

\subsection{Energy estimate and Lyapunov structure.}

Setting $\mathcal{H}=|\cdot|^{2}$, the following result states that $\mathbf{A} \mathbf{1}$ is true.

Lemma 4.2 (The Lyapunov structure). Assume H0'. There exist $C_{1}$ and a family $\left(C_{\alpha}^{\prime}\right)_{\alpha}$ only depending on $\varepsilon, \eta, \sigma, B_{1}$ and $D$ such that

$$
\left\{\begin{aligned}
\mathbb{E}\left(\left|u\left(t, W, u_{0}\right)\right|^{2}\right) & \leq e^{-\varepsilon \mu_{1} t}\left|u_{0}\right|^{2}+C_{1}, \\
\mathbb{E}\left(e^{-\alpha \tau}\left|u\left(\tau, W, u_{0}\right)\right|^{2} 1_{\tau<\infty}\right) & \leq\left|u_{0}\right|^{2}+C_{\alpha}^{\prime},
\end{aligned}\right.
$$

for any $t \geq 0$, any $\alpha>0$ and any stopping time $\tau$.

Proof of Lemma 4.2

We set $u=u\left(\cdot, s, W, u_{0}\right)$. We apply Ito Formula to $|u|^{2}$

$$
d|u|^{2}+2 \varepsilon\|u\|^{2} d t+2 \eta|u|_{2 \sigma+2}^{2 \sigma+2} d t=d M^{\prime}+\|\phi(u)\|_{\mathcal{L}_{2}(U ; H)}^{2} d t,
$$

where

$$
d M^{\prime}=2(u, \phi(u) d W) .
$$

Hence, taking into account Ho', we deduce

$$
d|u|^{2}+2 \varepsilon\|u\|^{2} d t+2 \eta|u|_{2 \sigma+2}^{2 \sigma+2} d t=d M^{\prime}+B_{1} d t .
$$

Now, we are able to deduce Lemma 4.2 from (4.5) by applying the same argument we used to deduce Lemma 3.5 from (3.5).

The next result will be useful in the following.

Lemma 4.3 (Exponential estimate for the growth of the solution). Assume that HO holds. There exists $\gamma_{2}>0$ only depending on $\varepsilon, \eta, \sigma, B_{1}$ and $D$ such that

$$
\mathbb{E}\left(\exp \left(\gamma_{2} \sup _{t \geq 0}\left(E_{u\left(\cdot, W, u_{0}\right)}(t)-B_{1} t\right)\right)\right) \leq 2 e^{\gamma_{2}\left|u_{0}\right|^{2}},
$$

for any $u_{0} \in H$. 
Proof of Lemma 4.3

We set for any $\gamma>0$

$$
\mathcal{M}_{\gamma}^{\prime}(t)=M^{\prime}(t)-\frac{\gamma}{2}\left\langle M^{\prime}\right\rangle(t)
$$

Remarking that

$$
d\left\langle M^{\prime}\right\rangle=4\left|\phi(u)^{*} u\right|^{2} d t \leq 4 c B_{1}\|u\|^{2} d t,
$$

and setting $\gamma_{1}=\frac{\nu}{2 c B_{1}}$, we deduce from (4.5) that

$$
E_{u}(t) \leq \mathcal{M}_{\gamma_{1}}(t)+\left|u_{0}\right|^{2}+B_{1} t .
$$

Now, we are able to deduce Lemma 4.3 from (4.7) by applying the same argument we used to deduce Lemma 3.6 from (3.6).

\subsection{Construction of the auxiliary process.}

Now, we build the auxiliary process $\widetilde{u}$ such that assumptions A2,..,A5 are true. This will allow to deduce Theorem 4.1 from Theorem 2.1

Let $K$ be a positive number. We set

$$
F(u)=(\varepsilon+\mathrm{i}) A u+(\eta+\lambda \mathrm{i})|u|^{2 \sigma} u,
$$

and

$$
\begin{array}{r}
\delta(u, \widetilde{u})=P_{N}\left((\eta+\lambda \mathrm{i})\left(|u|^{2 \sigma} u-|\widetilde{u}|^{2 \sigma} \widetilde{u}\right)+K\left|P_{N} \widetilde{u}\right|^{2 \sigma} P_{N}(\widetilde{u}-u)\right) \\
+L\left(1+|\widetilde{u}|_{H}^{2 \sigma}+|u|_{H}^{2 \sigma}\right) P_{N}(\widetilde{u}-u) .
\end{array}
$$

We now consider the following equation

$$
\left\{\begin{aligned}
d \widetilde{u}+F(\widetilde{u}) d t+\delta\left(u\left(t, W, u_{0}\right), \widetilde{u}\right) d t & =\phi(\widetilde{u}) d W, \\
\widetilde{u}(0) & =\widetilde{u}_{0} .
\end{aligned}\right.
$$

It is not difficult to deduce from $\mathbf{H O}$ that there exists a unique $H$-valued solution to (3.12) when $\left(u_{0}, \widetilde{u}_{0}\right) \in H^{2}$. Moreover, $\widetilde{u}$ is a non anticipative measurable map of $W$ (See Remark [1.2). It follows from the uniqueness that $(u, \widetilde{u})$ is a homogenous weak Markov process. Moreover, its law $\mathcal{D}(u, \widetilde{u})$ is measurably depending of its initial condition $\left(u_{0}, \widetilde{u}_{0}\right)$.

We will denote the dependance of $(u, \widetilde{u})$ with respect to $\left(t, W, u_{0}, \widetilde{u}_{0}\right)$ as follows

$$
(u(t), \widetilde{u}(t))=\left(u\left(t, W, u_{0}\right), \widetilde{u}\left(t, W, u_{0}, \widetilde{u}_{0}\right)\right),
$$

and we deduce A0.

Taking into account $\mathbf{H} \mathbf{1}$ and the uniqueness of the solution of (4.2) under H0', we deduce $\mathbf{A} \mathbf{3}$ by setting

$$
h(u, \widetilde{u})=-g(\widetilde{u}) \delta(u, \widetilde{u}) .
$$

We first state a Lemma that will be useful in the following.

Lemma 4.4 (Exponential estimate for the growth of the auxiliary process). Assume H0'. There exists $C, B, K_{L}, \gamma_{0}^{\prime}, \gamma_{0}>0$ not depending on $N$ such that if $K=K_{L}$

$$
\mathbb{E}\left(\exp \left(\gamma_{0}^{\prime} \sup _{t \geq 0}\left(E_{u\left(\cdot, W, u_{0}\right)}(t)+E_{\widetilde{u}\left(\cdot, W, \widetilde{u}_{0}, u_{0}\right)}(t)-B t\right)\right)\right) \leq C e^{\gamma_{0}\left(\left|u_{0}\right|^{2}+\left|\widetilde{u}_{0}\right|^{2}\right)},
$$


Exponential Mixing for Stochastic PDEs: The Non-Additive Case.

for any $\left(u_{0}, \widetilde{u}_{0}\right) \in H^{2}$.

Proof of Lemma 4.4

For any function $f$, we denote by $\delta f(u)$ the value $f(\widetilde{u})-f(u)$. Moreover, we set $r=\widetilde{u}-u$.

Taking the Ito Formula of $|\widetilde{u}|^{2}$, we obtain

$$
\begin{aligned}
d|\widetilde{u}|^{2}+ & 2 \varepsilon\|\widetilde{u}\|^{2} d t+2 \eta|\widetilde{u}|_{2 \sigma+2}^{2 \sigma+2} d t+2 K\left|P_{N} \widetilde{u}\right|_{2 \sigma+2}^{2 \sigma+2} d t=2(\widetilde{u}, \phi(\widetilde{u}) d W) \\
& +\|\phi(u)\|_{\mathcal{L}_{2}(U ; H)}^{2} d t+I(t) d t+2\left(P_{N} \widetilde{u},(\eta+\lambda \mathrm{i}) \delta\left(|u|^{2 \sigma} u\right)\right) d t,
\end{aligned}
$$

where $I=I_{1}+I_{2}$ and

$$
\begin{aligned}
& I_{1}(t)=-2 L\left(1+|\widetilde{u}|^{2 \sigma}+|u|^{2 \sigma}\right)\left(P_{N} \widetilde{u}, P_{N} r\right), \\
& I_{2}(t)=2 K\left(\left|P_{N} \widetilde{u}\right|^{2 \sigma} P_{N} \widetilde{u}, P_{N} u\right) .
\end{aligned}
$$

Applying arithmetico-geometric inequalities, we obtain

$$
I_{2}(t) \leq K\left|P_{N} \widetilde{u}\right|_{2 \sigma+2}^{2 \sigma+2}+c K|u|_{2 \sigma+2}^{2 \sigma+2} .
$$

Remarking that

$$
\left(P_{N} \widetilde{u}, P_{N} r\right)=\left|P_{N} \widetilde{u}\right|^{2}-\left(P_{N} \widetilde{u}, P_{N} u\right),
$$

we deduce from Schwarz inequality

$$
I_{1}(t) \leq 2 L\left(1+|\widetilde{u}|^{2 \sigma}+|u|^{2 \sigma}\right)\left|P_{N} \widetilde{u}\right|\left|P_{N} u\right|,
$$

which implies by applying an arithmetico-geometric inequality

$$
I_{1}(t) \leq 1+\frac{K_{L}}{2}\left|P_{N} \widetilde{u}\right|_{2 \sigma+2}^{2 \sigma+2}+\frac{\eta}{2}\left(|u|_{2 \sigma+2}^{2 \sigma+2}+|\widetilde{u}|_{2 \sigma+2}^{2 \sigma+2}\right) .
$$

Applying arithmetico-geometric inequality, it follows

$$
2\left(P_{N} \widetilde{u},(\eta+\lambda \mathrm{i}) \delta\left(|u|^{2 \sigma} u\right)\right) \leq \frac{K_{L}}{2}\left|P_{N} \widetilde{u}\right|_{2 \sigma+2}^{2 \sigma+2}+\frac{\eta}{2}\left(|u|_{2 \sigma+2}^{2 \sigma+2}+|\widetilde{u}|_{2 \sigma+2}^{2 \sigma+2}\right) .
$$

Combining (4.5), 4.6), (4.11), (4.12), (4.13), 4.14) and HO', we obtain that if $K=K_{L}$

$$
d E_{\widetilde{u}}+4 c_{1}(1+2 K) d E_{u} \leq \mathcal{M}(t)+B t,
$$

where $\mathcal{M}$ has been defined as in Section 3.2

$M(t)=4 c_{1}(1+2 K) M^{\prime}(t)+2 \int_{s}^{t}(u(r), \phi(u(r)) d W(r)), \quad \mathcal{M}(t)=M(t)-\frac{\gamma_{0}}{2}\langle M\rangle(t)$.

Now, since there is no loss of generality of assuming $c_{1} \geq 1$, we are able to deduce Lemma 4.4 from (4.15) by applying the same argument we used to deduce Lemma 3.6 from (3.6).

We now fix $K=K_{L}$ and we state a result analogous to Proposition 3.7

Proposition 4.5. Assume that H0' holds. There exist $\left(C_{N}\right)_{N}, \alpha, \gamma>0, \gamma^{\prime} \in(0,1]$ and $N_{0}$ depending only on $B_{1}, L, \varepsilon, \eta$ and $D$ such that for any $N \geq N_{0}$ and any $\left(t, u_{0}, \widetilde{u}_{0}\right) \in(0, \infty) \times H^{2}$

$$
\mathbb{E}\left(\left(e^{\frac{\varepsilon \mu_{1}}{8} t}|r(t)|^{2}+\int_{0}^{t} e^{\frac{\varepsilon \mu_{1}}{8} s} Z(s) d s\right)^{\gamma^{\prime}}\right) \leq C_{N}|r(0)|^{2 \gamma^{\prime}} e^{\gamma\left(\left|u_{0}\right|^{2}+\left|\widetilde{u}_{0}\right|^{2}\right)},
$$


Exponential Mixing for Stochastic PDEs: The Non-Additive Case.

where

$$
\left\{\begin{aligned}
r & =\widetilde{u}-u, \quad \widetilde{u}=\widetilde{u}\left(\cdot, W, u_{0}, \widetilde{u}_{0}\right), \quad u=u\left(\cdot, W, u_{0}\right), \quad \beta=4 \sigma+\frac{2 \sigma+2}{2-\sigma} \\
Z & =\left(\|u\|^{2}+\|\widetilde{u}\|^{2}+|u|_{2 \sigma+2}^{2 \sigma+2}+|\widetilde{u}|_{2 \sigma+2}^{2 \sigma+2}\right)|r|^{2}+\left(1+|u|^{\beta}+|\widetilde{u}|^{\beta}\right)\|r\|^{2} .
\end{aligned}\right.
$$

By Chebyshev inequality, A2 immediately follows.

Proof of Proposition 4.5

For any function $f$, we denote by $\delta f(u)$ the value $f(\widetilde{u})-f(u)$. Taking the difference between (4.2) and (4.9), we obtain

$$
\begin{aligned}
d r+(\varepsilon+\mathrm{i}) A r d t+L\left(1+|\widetilde{u}|_{H}^{2 \sigma}+|u|_{H}^{2 \sigma}\right) & P_{N} r d t+K P_{N}\left(\left|P_{N} \widetilde{u}\right|^{2 \sigma} P_{N} r\right) d t \\
= & \delta \phi(u) d W-(\eta+\lambda \mathrm{i}) Q_{N} \delta\left(|u|^{2 \sigma} u\right) d t .
\end{aligned}
$$

Hence, applying Ito Formula to $|r|^{2}$, we have

$$
\begin{array}{r}
d|r|^{2}+2 \varepsilon\|r\|^{2} d t+2 L\left(1+|\widetilde{u}|^{2 \sigma}+|u|^{2 \sigma}\right)\left|P_{N} r\right|^{2} d t \leq 2(r, \delta \phi(u) d W) \\
-2\left(Q_{N} r,(\eta+\lambda \mathrm{i}) \delta\left(|u|^{2 \sigma} u\right)\right) d t+\|\delta \phi(u)\|_{\mathcal{L}_{2}(U ; H)}^{2} d t .
\end{array}
$$

Remarking that for any $(x, y) \in \mathbb{C}^{2}$

$$
\left.|| x\right|^{2 \sigma} x-|y|^{2 \sigma} y\left|\leq C_{\sigma}\left(|x|^{2 \sigma}+|y|^{2 \sigma}\right)\right| x-\left.y\right|^{2},
$$

it follows from Hölder inequality

$$
-\left(Q_{N} r,(\eta+\lambda \mathrm{i}) \delta\left(|u|^{2 \sigma} u\right)\right) \leq c\left|Q_{N} r\right|_{2 \sigma+2}|r|_{2 \sigma+2}\left(|\widetilde{u}|_{2 \sigma+2}^{2 \sigma}+|u|_{2 \sigma+2}^{2 \sigma}\right) .
$$

Setting

$$
s_{0}=\frac{\sigma d}{2 \sigma+2}, \quad s_{+}=\frac{1}{\sigma+1},
$$

we deduce from Sobolev Embedding $H^{s_{0}}(D ; \mathbb{C}) \subset L^{2 \sigma+2}(D ; \mathbb{C})$ that

$$
-\left(Q_{N} r,(\eta+\lambda \mathrm{i}) \delta\left(|u|^{2 \sigma} u\right)\right) \leq c\left\|Q_{N} r\right\|_{s_{0}}\|r\|_{s_{0}}\left(|\widetilde{u}|_{2 \sigma+2}^{2 \sigma}+|u|_{2 \sigma+2}^{2 \sigma}\right) .
$$

Remarking that $s_{0}<s_{+}<1$ and that $\left\|Q_{N} r\right\|_{s_{+}} \leq \mu_{N+1}^{\frac{s_{+}-s_{0}}{2}}\left\|Q_{N} r\right\|_{s_{0}}$, we deduce from interpolatory inequality

$$
-\left(Q_{N} r,(\eta+\lambda \mathrm{i}) \delta\left(|u|^{2 \sigma} u\right)\right) \leq c \mu_{N+1}^{-\frac{s_{+}-s_{0}}{2}}\|r\|^{2 s_{+}}|r|^{2\left(1-s_{+}\right)}\left(|\widetilde{u}|_{2 \sigma+2}^{2 \sigma}+|u|_{2 \sigma+2}^{2 \sigma}\right) .
$$

Hence, it follows from arithmetico-geometric inequality that there exists $\alpha \in(0,1)$ only depending on $\sigma$ and $d$ such that

$$
-\left(Q_{N} r,(\eta+\lambda \mathrm{i}) \delta\left(|u|^{2 \sigma} u\right)\right) \leq \frac{\varepsilon}{2}\|r\|^{2}+\frac{c}{\mu_{N+1}^{\alpha}}|r|^{2}\left(|\widetilde{u}|_{2 \sigma+2}^{2 \sigma+2}+|u|_{2 \sigma+2}^{2 \sigma+2}\right) .
$$

Recall Ho',

$$
\|\delta \phi(u)\|_{\mathcal{L}_{2}(U ; H)}^{2} \leq L\left(1+|\widetilde{u}|^{2 \sigma}+|u|^{2 \sigma}\right)|r|^{2} .
$$

Remarking that

$$
|r|^{2} \leq\left|P_{N} r\right|^{2}+\frac{1}{\mu_{N+1}^{s_{+}}}\left\|Q_{N} r\right\|_{s_{+}}^{2}
$$


Exponential Mixing for Stochastic PDEs: The Non-Additive Case.

and making interpolatory inequality analogous to those done to obtain (4.18), we obtain

$$
\begin{aligned}
\|\delta \phi(u)\|_{\mathcal{L}_{2}(U ; H)}^{2} \leq \frac{\varepsilon}{4}\|r\|^{2}+\frac{c}{\mu_{N+1}^{\alpha}}|r|^{2}\left(|\widetilde{u}|_{2 \sigma+2}^{2 \sigma+2}+|u|_{2 \sigma+2}^{2 \sigma+2}\right) \\
+L\left(1+|\widetilde{u}|^{2 \sigma}+|u|^{2 \sigma}\right)\left|P_{N} r\right|^{2} .
\end{aligned}
$$

Hence, setting

$$
V(t)=1+|\widetilde{u}(t)|_{2 \sigma+2}^{2 \sigma+2}+|u(t)|_{2 \sigma+2}^{2 \sigma+2},
$$

we deduce from (4.16), (4.18) and (4.19) that there exists $\Lambda_{1}>0$ such that

$$
\begin{aligned}
d|r|^{2}+\left(\frac{5}{4} \varepsilon\|r\|^{2}-\frac{\Lambda_{1}}{\mu_{N+1}^{\alpha}} V|r|^{2}+L\left(1+|\widetilde{u}|^{2 \sigma}+|u|^{2 \sigma}\right)\right. & \left.\left|P_{N} r\right|^{2}\right) d t \\
& \leq 2(r, \delta \phi(u) d W) .
\end{aligned}
$$

Taking into account (4.15), Ito Formula of $\left(\mu_{N+1}^{\alpha}+4 c_{1}(1+2 K)|u|^{2}+|\widetilde{u}|^{2}\right)|r|^{2}$ gives

$$
\begin{aligned}
d\left(X|r|^{2}\right)+\left(\frac{\varepsilon \mu_{1}}{4}-\frac{\Lambda_{1}}{\mu_{N+1}^{\alpha}} V\right) X|r|^{2} d t & +\varepsilon X\|r\|^{2} d t+Y|r|^{2} d t \\
& \leq d M_{1}+B|r|^{2} d t+d\left\langle M, M_{r}\right\rangle .
\end{aligned}
$$

where

$$
\left\{\begin{aligned}
M_{r}(t) & =\int_{0}^{t}(r, \delta \phi(u) d W), \\
X(t) & =\mu_{N+1}^{\alpha}+4 c_{1}(1+2 K)|u(t)|^{2}+|\widetilde{u}(t)|^{2}, \\
Y(t) & =\mu_{N+1}^{\alpha}+4 c_{1}(1+2 K)\left(\varepsilon\|u(t)\|^{2}+\eta|u(t)|_{2 \sigma+2}^{2 \sigma+2}\right)+\left(\varepsilon\|\widetilde{u}(t)\|^{2}+\eta|\widetilde{u}(t)|_{2 \sigma+2}^{2 \sigma+2}\right), \\
d M_{1} & =X d M_{r}+|r|^{2} d M .
\end{aligned}\right.
$$

Notice that

$$
d\left\langle M, M_{r}\right\rangle \leq c\left(\left|\phi^{*}(u) u\right|+\left|\phi^{*}(\widetilde{u}) \widetilde{u}\right|\right)\left|\delta \phi^{*} r\right| d t
$$

It follows from Ho' that

$$
d\left\langle M, M_{r}\right\rangle \leq c(|u|+|\widetilde{u}|)|r|^{2}\left(1+|u|^{\sigma}+|\widetilde{u}|^{\sigma}\right),
$$

which yields

$$
B|r|^{2} d t+d\left\langle M, M_{r}\right\rangle \leq \frac{c}{\mu_{N+1}^{\alpha}} V|r|^{2} d t \leq \frac{c}{\mu_{N+1}^{\alpha}} V X|r|^{2} d t .
$$

It follows that

$$
d\left(X|r|^{2}\right)+\left(\frac{\varepsilon \mu_{1}}{4}-\frac{\Lambda_{2}}{\mu_{N+1}^{\alpha}} V\right)\left(X|r|^{2}\right) d t+\varepsilon X\|r\|^{2} d t+Y|r|^{2} d t \leq d M_{1} .
$$

Integrating this formula and taking the expectation, it follows

$\mathbb{E}\left(\begin{array}{l}e^{\frac{\varepsilon \mu_{1}}{8} t} G(t)^{-1} X(t)|r(t)|^{2}+ \\ \int_{0}^{t} e^{\frac{\varepsilon \mu_{1}}{8} s} G(s)^{-1}\left(\varepsilon X(s)\|r(s)\|^{2}+Y(s) e^{\frac{\Lambda}{\mu_{N+1}^{\alpha}}\left(|u(s)|^{2}+|\widetilde{u}(s)|^{2}\right)}|r(s)|\right) d s\end{array}\right) \leq|r(0)|^{2}$,

where

$$
G(t)=e^{-\frac{\varepsilon \mu_{1}}{8} t+\frac{\Lambda}{\mu_{N+1}^{\alpha}}\left(E_{u}(t)+E_{\tilde{u}}(t)\right)} .
$$


Notice that $1+|u|^{\beta}+|\widetilde{u}|^{\beta} \leq C_{N} Y e^{\frac{\Lambda}{\mu_{N+1}^{\alpha}}\left(|u|^{2}+|\widetilde{u}|^{2}\right)}$. Hence, choosing $N$ sufficiently high and $\varepsilon>0$ sufficiently small, we are able to deduce Proposition 4.5 from Lemma 4.4 as we have deduced Proposition 3.7 from Lemma 3.6

To end the proof of Theorem 4.1 we establish that

$$
|h(u, \widetilde{u})|^{2} \leq C_{N} Z .
$$

Hence, by Chebyshev inequality, we deduce A4 and A5 from Proposition 4.5 .

Proof of 4.20)

Taking into account $\mathbf{H 1}$ and 4.10 , we remark that it is sufficient to establish

$$
|\delta(\widetilde{u}, u)|^{2} \leq C_{N} Z \text {. }
$$

Recalling (4.8), we deduce that it is sufficient to estimate the three following values

$$
\begin{aligned}
& I_{1}=\left|P_{N}\left(|u|^{2 \sigma} u-|\widetilde{u}|^{2 \sigma} \widetilde{u}\right)\right|^{2}, \\
& I_{2}=\left|P_{N}\left(\left|P_{N} \widetilde{u}\right|^{2 \sigma} P_{N} r\right)\right|^{2}, \\
& I_{3}=\left(1+|\widetilde{u}|^{4 \sigma}+|u|^{4 \sigma}\right)\left|P_{N} r\right|^{2} .
\end{aligned}
$$

For $I_{3}$, the result is obvious. Applying successively Hölder inequality and the equivalence of the norm in finite-dimensional spaces, it follows

$$
I_{2}=\left|P_{N}\left(\left|P_{N} \widetilde{u}\right|^{2 \sigma} P_{N} r\right)\right|^{2} \leq\left|P_{N} \widetilde{u}\right|_{4 \sigma+2}^{4 \sigma}\left|P_{N} r\right|_{4 \sigma+2}^{2} \leq K_{N}^{\prime}|\widetilde{u}|^{4 \sigma}|r|^{2} \leq C_{N} Z .
$$

The equivalence of the norm in finite-dimensional spaces gives

$$
I_{1}=\left|P_{N}\left(|u|^{2 \sigma} u-|\widetilde{u}|^{2 \sigma} \widetilde{u}\right)\right|^{2} \leq\left. K_{N}^{\prime}|| u\right|^{2 \sigma} u-\left.|\widetilde{u}|^{2 \sigma} \widetilde{u}\right|_{1} ^{2} .
$$

Hence, we deduce from (4.17) that

$$
I_{1} \leq K_{N}^{\prime}\left|\left(|u|^{2 \sigma}+|\widetilde{u}|^{2 \sigma}\right) r\right|_{1}^{2} .
$$

We first treat the case $\sigma \leq 1$. In that case, a Schwarz inequality gives

$$
I_{1} \leq K_{N}^{\prime}\left(|u|_{4 \sigma}^{4 \sigma}+|\widetilde{u}|_{4 \sigma}^{4 \sigma}\right)|r|^{2} \leq C_{N} Z
$$

Now, we treat the case $\sigma \in(1,2)$. In that case $d=1$. Sobolev Embedding $H^{1}(D) \subset L^{\infty}(D)$ gives

$$
I_{1} \leq K_{N}^{\prime}\left(|u|_{2 \sigma}^{4 \sigma}+|\widetilde{u}|_{2 \sigma}^{4 \sigma}\right)\|r\|^{2}
$$

Sobolev Embedding $H^{\frac{\sigma-1}{2 \sigma}}(D) \subset L^{2 \sigma}(D)$ and interpolatory inequality gives

$$
|u|_{2 \sigma}^{4 \sigma} \leq\|u\|_{\frac{\sigma-1}{2 \sigma}}^{4 \sigma} \leq\|u\|^{2 \sigma-2}|u|^{2 \sigma+2} \leq\|u\|^{2}+|u|^{\frac{2 \sigma+2}{2-\sigma}},
$$

which yields

$$
I_{1} \leq C_{N} Z
$$

So we have $I_{i} \leq C_{N} Z$ for $i=1,2,3$, which yields 4.20). 
Exponential Mixing for Stochastic PDEs: The Non-Additive Case.

\subsection{Conclusion and Remarks.}

We have proved that assumptions of Theorem 2.1 are verified. So Theorem 4.1 follows.

Actually, A0 is consequence of the well-posedness of equations.

We set $\mathcal{H}=|\cdot|^{2}$. As shown in Lemma 4.2 we have $\mathbf{A} \mathbf{1}$.

Assumption $\mathbf{A} 3$ has been proved at the beginning of section 4.3.

We deduce A2 directly from Proposition 4.5] and A4, A5 from (4.20) and Proposition 4.5 .

Since A0, ., A5 are established, we can apply Theorem 2.1] which yields Theorem 4.1 .

Remark 4.6. Notice that the proof Proposition 4.5 is much more difficult than the proof of Proposition 3.7 because it involves energies control of both $u_{1}$ and $\widetilde{u}$. Then, in order to apply it, we have to establish energy estimate of both $u_{1}$ and $\widetilde{u}$ (Lemma 4.4). That is the reason why the building of the auxiliary process is more complicated than for NS or for CGL with a global Lipschitz noise. For instance, if we set

$$
\delta\left(\widetilde{u}, u_{1}\right)=K P_{N}\left(\widetilde{u}-u_{1}\right)
$$

then we would have an energy Lemma on $\widetilde{u}$ analogous to Lemma 4.4 and a Proposition analogous to Proposition 4.5 under a truncation condition on $\widetilde{u}$. The problem is that we do not know if it is possible to choose $K$ and $N$ such that both result are true. So we can not combine them.

Remark 4.7. There exists a lot of variations of Theorem 4.1. Hence (4.3) could be strengthened into

$$
\int_{H} \exp \left(\alpha_{1}\left(B_{1}, \varepsilon, \eta, D\right)|u|^{2}\right) d \mu(u)<\infty .
$$

Moreover, one can work in $H_{0}^{1}(D ; \mathbb{C})$ in the defocusing case under the $H^{1}$-subcritical condition $(d-2) \sigma<2$ and in the focusing case under the subcritical condition $\sigma<\frac{2}{d}$. In the focusing case, the rate of convergence is greater than any power of time instead of being exponential in time.

In $\mathbf{H 1}$, the boundedness of $g$ could be replaced by the existence of $C$ such that

$$
|g(u)|_{\mathcal{L}(H ; U)} \leq C \exp \left(\frac{\varepsilon \mu_{1}}{4}|u|^{2}\right) .
$$

Contrary to Navier-Stokes, the coefficient in the exponential cannot be as high as we want because it seems that for the locally Lipschitz CGL, there is no property analogous to Proposition 3.7

In H0', the boundedness of $\phi$ could be replaced by $|\phi(u)| \leq C\left(1+|u|^{\gamma}\right)$. If $\gamma \leq \sigma$, then the rate of convergence remains exponential. If $\gamma<\sigma+1$, then the rate of convergence becomes greater than any power of time instead of being exponential in time. Moreover for any $p$ there exists $c_{p}$ such that if there exists $C$ such that $|\phi(u)| \leq C+c_{p}|u|^{\sigma+1}$, then the rate of convergence is greater than $(1+t)^{-p}$.

\section{REFERENCES}

[1] M. Barton-Smith, Invariant measure for the stochastic Ginzburg Landau equation, Nonlinear Differential Equations Appl. 11 , no. 1, 29-52, 2004.

[2] P. Bebouche, A. Jüngel, Inviscid limits of the Complex Ginzburg-Landau Equation, Commun. Math. Phys. 214, 201-226, 2000. 
Exponential Mixing for Stochastic PDEs: The Non-Additive Case.

[3] J. Bricmont, A. Kupiainen and R. Lefevere, Exponential mixing for the 2D stochastic NavierStokes dynamics, Commun. Math. Phys. 230, No.1, 87-132, 2002.

[4] P. Constantin and C. Foias, Navier-Stokes Equations, University of Chicago Press, Chicago, IL, 1988.

[5] G. Da Prato, A. Debussche, Ergodicity for the 3D stochastic Navier-Stokes equations J. Math. Pures Appl. (9) 82 (2003), no. 8, 877-947.

[6] G. Da Prato and J. Zabczyk, Stochastic equations in infinite dimensions, Encyclopedia of Mathematics and its Applications, Cambridge University Press, 1992.

[7] G. Da Prato and J. Zabczyk, Ergodicity for Infinite Dimensional Systems, London Mathematical Society Lecture Notes, n.229, Cambridge University Press, 1996.

[8] A. Debussche, C. Odasso, Ergodicity for the weakly damped stochastic Non-linear Schrödinger equations, to appear in Journal of Evolution Equations.

[9] W. E, J.C. Mattingly, Y. G. Sinai, Gibbsian dynamics and ergodicity for the stochastically forced Navier-Stokes equation, Commun. Math. Phys. 224, 83-106, 2001.

[10] G.E. Falkovich, I. Kolokolov, V. Lebedev, S.K. Turitsyn, Self-focusing in the perturbed and unperturbed nonlinear Schroedinger equation in critical dimension, J. Appl. Math. 60, 183-240 (2000).

[11] F. Flandoli and B. Maslowski, Ergodicity of the 2-D Navier-Stokes equation under random perturbations, Commun. Math. Phys. 171, 119-141, 1995.

[12] V. Ginzburg, L. Landau, On the theorie of superconductivity, Zh. Eksp. Fiz. 20, 1064(1950) English transl. in: Men of Physics: L.D. Landau. Vol. I. Ter Haar (ed.). New York: Pergammon Press. 1965. pp. 546-568

[13] O. Goubet, Regularity of the attractor for a weakly damped nonlinear Schrödinger equation, Appl. Anal. 60, No.1-2, 99-119, 1996.

[14] M. Hairer, Exponential Mixing Properties of Stochastic PDEs Through Asymptotic Coupling, Proba. Theory Related Fields, 124, 3 :345-380, 2002.

[15] M. Hairer, J. Mattingly, Ergodicity of the 2D Navier-Stokes equations with degenerate forcing, preprint.

[16] G. Huber, P. Alstrom, Universal Decay of vortex density in two dimensions, Physica A 195, 448-456, 1993.

[17] S. Kuksin, On exponential convergence to a stationary mesure for nonlinear PDEs, The M. I. Viishik Moscow PDE seminar, Amer. Math. Soc. Trans. (2), vol 206, Amer. Math. Soc., 2002 .

[18] S. Kuksin and A. Shirikyan, Stochastic dissipative PDE's and Gibbs measures, Comm. Math. Phys. 213 (2000), no. 2, 291-330.

[19] S. Kuksin, A. Shirikyan, Stochastic dissipative PDE's and Gibbs measures, Commun. Math. Phys. 213, 291-330, 2000.

[20] S. Kuksin and A. Shirikyan,Ergodicity for the randomly forced $2 D$ Navier-Stokes equations, Math. Phys. Anal. Geom. 4, 2001.

[21] S. Kuksin, A. Shirikyan, A coupling approach to randomly forced randomly forced PDE's I, Commun. Math. Phys. 221, 351-366, 2001.

[22] S. Kuksin, A. Piatnitski, A. Shirikyan, A coupling approach to randomly forced randomly forced PDE's II, Commun. Math. Phys. 230, No.1, 81-85, 2002.

[23] S. Kuksin, A. Shirikyan, Coupling approach to white-forced nonlinear PDEs, J. Math. Pures Appl. 1 (2002) pp. 567-602.

[24] S. Kuksin, A. Shirikyan, Randomly forced CGL equation: Stationnary measure and the inviscid limit, J. Phys. A 37, no. 12, 3805-38222004.

[25] T. Lindvall, Lectures on the coupling method, John Wiley and Sons, New York, 1992.

[26] J. Mattingly, Exponential convergence for the stochastically forced Navier-Stokes equations and other partially dissipative dynamics, Commun. Math. Phys. 230, 421-462, 2002.

[27] J. Mattingly, E. Pardoux, Ergodicity of the 2D Navier-Stokes Equations with Degenerate Stochastic Forcing, preprint 2004.

[28] A. Newel, J. Whitehead, Finite bandwidth, finite amplitude convection, J. Fluid Mech. 38, 279-303, 1969.

[29] A. Newel, J. Whitehead, Review of the finite bandwidth concept, H. Leipholz. editor. Proceedings of the Internat. Union of Theor. and Appl. Math.. Berlin: Springer. 1971, pp 284-289.

[30] C. Odasso, Ergodicity for the stochastic Complex Ginzburg-Landau equations, to appear in Annales de l'institut Henri-Poincar, Probabilits et Statistiques. 
Exponential Mixing for Stochastic PDEs: The Non-Additive Case.

[31] A. Shirikyan, Exponential mixing for 2D Navier-Stokes equation pertubed by an unbounded noise, J. Math. Fluid Mech. 6, no. 2, 169-193, 2004.

[32] R. Temam, Navier-Stokes Equations. Theory and Numerical Analysis., North-Holland, Amsterdam-New York-Oxford,1977. 